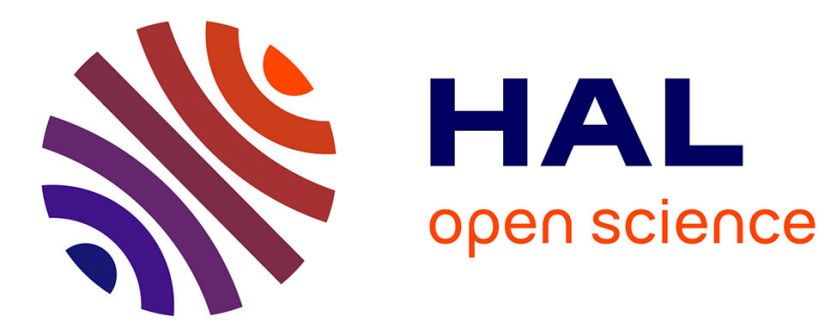

\title{
Effects of radiation in turbulent channel flow: analysis of coupled direct numerical simulations
}

\author{
Ronan Vicquelin, Yufang Zhang, Olivier Gicquel, Jean Taine
}

\section{To cite this version:}

Ronan Vicquelin, Yufang Zhang, Olivier Gicquel, Jean Taine. Effects of radiation in turbulent channel flow: analysis of coupled direct numerical simulations. Journal of Fluid Mechanics, 2014, 10.1017/jfm.2014.368 . hal-01232488

\section{HAL Id: hal-01232488 \\ https://hal.science/hal-01232488}

Submitted on 23 Nov 2015

HAL is a multi-disciplinary open access archive for the deposit and dissemination of scientific research documents, whether they are published or not. The documents may come from teaching and research institutions in France or abroad, or from public or private research centers.
L'archive ouverte pluridisciplinaire HAL, est destinée au dépôt et à la diffusion de documents scientifiques de niveau recherche, publiés ou non, émanant des établissements d'enseignement et de recherche français ou étrangers, des laboratoires publics ou privés. 


\title{
Effects of radiation in turbulent channel flow: Analysis of coupled direct numerical simulations
}

\author{
R. Vicquelin ${ }^{1,2} \dagger$, Y. F. Zhang ${ }^{1,2} \dagger$ ，O. Gicquel ${ }^{1,2}$ ， and J. Taine ${ }^{1,2}$ \\ ${ }^{1}$ CNRS, UPR 288 Laboratoire d'Energétique Moléculaire et Macroscopique, Combustion \\ (EM2C), Grande Voie des Vignes, 92295 Châtenay-Malabry, France \\ ${ }^{2}$ Ecole Centrale Paris, Grande Voie des Vignes, 92295 Châtenay-Malabry, France \\ (Received ?; revised ?; accepted ?. - To be entered by editorial office)
}

The role of radiative energy transfer on turbulent boundary layers is carefully analyzed, focusing on the effect on temperature fluctuations and turbulent heat flux. The study is based on direct numerical simulations of channel flows with hot and cold walls coupled to a Monte-Carlo method to compute the field of radiative power. In the studied conditions, the structure of the boundary layers is strongly modified by radiation. Temperature fluctuations and turbulent heat flux are reduced, and new radiative terms appear in their respective balance equations. It is shown that they counteract turbulence production terms. These effects are analyzed under different conditions of Reynolds number and wall temperature. It is shown that collapsing of wall-scaled profiles is not efficient when radiation is considered. This drawback is corrected by the introduction of a radiationbased scaling. Finally, the significant impact of radiation on turbulent heat transfer is studied in terms of turbulent Prandtl number. A model for this quantity, based on the new proposed scaling, is developed and validated.

Key words: Direct Numerical Simulation; Turbulence; Radiative transfer; Monte-Carlo simulation; Channel flow; Turbulent heat transfer

\section{Introduction}

Radiation plays an important role in many industrial applications, particularly in combustion systems such as boilers, gas turbines, rocket engines and furnaces. For instance, in gas turbines, a crucial part of the heat transferred from hot gas to the combustor solid walls comes from radiative energy transfer (Lefebvre \& Ballal 2010). The importance of radiation is even higher in modern gas turbines as the pressure ratio increases, which makes the cooling of combustor walls more difficult. Moreover, radiation can influence the temperature distribution and hence the emission of pollutants in combustion systems. Therefore, an accurate prediction of radiation effects is important for the design of combustors.

Among the studies of radiation effects in turbulent flows, much attention has been given to the interaction between turbulence and radiation (TRI). Two aspects of TRI can be identified: the effects of radiation on the temperature and species concentrations

$\dagger$ Email address for correspondence: ronan.vicquelin@ecp.fr

$\ddagger$ Current address: AVIC Commercial Aircraft Engine Co., Ltd., Shanghai, 200241, P. R. China 

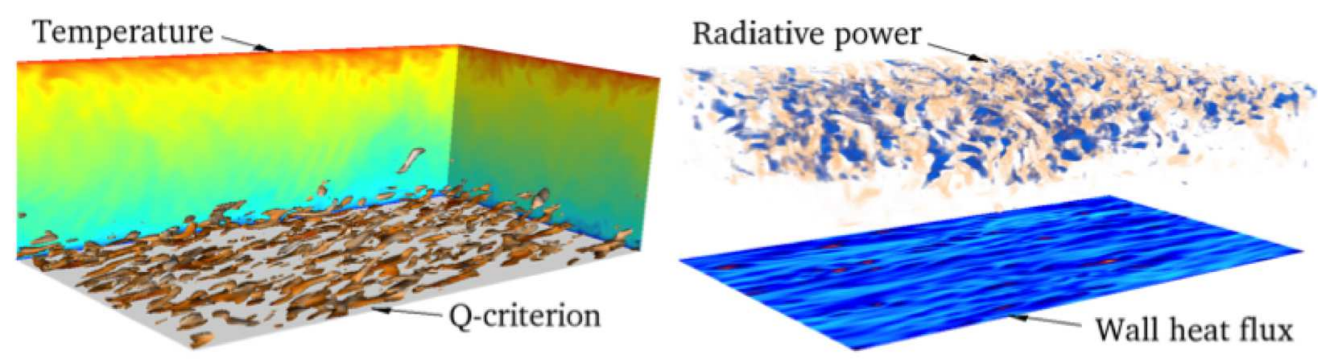

FiguRE 1. Snapshot of temperature (left, slices), turbulent eddies identified by the Q-criterion (left, near bottom wall only), wall heat flux (right, bottom wall only) and radiative power (right, volume rendering near the upper wall only).

turbulent fields and vice-versa. A comprehensive review about TRI can be found in Coelho $(2007,2012)$.

Regarding the effects of turbulence on radiation, it is observed that turbulence leads to an increase in medium transmissivity (Jeng \& Faeth 1984; Gore et al. 1987), radiative power (Coelho 2004; Tessé et al. 2004) and heat losses (Li \& Modest 2003; Tessé et al. 2004). Coelho et al. (2003) reported that, in a non-luminous turbulent jet diffusion flame, TRI enhanced the heat losses by a factor of $30 \%$ while a similar change in a luminous turbulent flame was also revealed by Tessé et al. (2004). Moreover, individual contributions to emission and absorption TRI have been isolated and quantified in a 1D premixed combustion system (Wu et al. 2005), a homogeneous isotropic non-premixed combustion system (Deshmukh et al. 2007) and a 1D turbulent non-premixed flame (Deshmukh et al. 2008). In the latter two studies involving coupled direct numerical simulations, the consistency of the radiative transfer solver with the order of spatial discretization error in the DNS solver has been improved by considering a high-order Monte-Carlo method from Wu et al. (2007). Gupta et al. (2009) have shown that the effects of temperature fluctuations on the mean radiative power in a non-reactive channel flow is negligible, while the presence of a turbulent flame that enhances temperature fluctuations makes TRI important. The prediction of such effects in Reynolds-averaged numerical simulations requires a specific modeling of the mean radiative power (Tessé et al. 2004; Haworth 2010). In large-eddy simulations, subgrid turbulence-radiation interactions are often neglected. A couple of studies (Gupta et al. 2013; Soucasse et al. 2014) have recently studied subfilter modeling of the radiative power.

By contrast to the former, only a few studies have been devoted to the effect of radiation on turbulence. Among them, Soufiani (1991) carried out a theoretical analysis of the influence of radiation on thermal turbulence spectra and it was concluded that radiation acted as a dissipation term and it could smooth the intensity of temperature fluctuations and modify the structure of the temperature variance spectrum. Damien et al. (2012) also reported that radiation modifies the level of temperature fluctuations and homogenizes the spectral distribution of energy. Moreover, it was reported that the Reynolds stress and turbulence structure in supersonic shear layers were modified by radiation (Ghosh et al. 2011).

The objective of this paper is to investigate the effects of radiation on the boundary layer structure of turbulent channel flows. It has already been reported by Zhang et al. (2013a) that radiation can significantly modify the mean temperature profile and consequently, the temperature wall law and the wall conductive heat flux. The different observed effects on the mean temperature profile have been explained by the antagonist behaviors of gas-wall and gas-gas radiative contributions. In the present study, Direct 
Numerical Simulations (DNS) from Zhang et al. (2013a) of channel flows coupled with a reciprocal Monte Carlo method to deal with radiation are analyzed. The Monte-Carlo method solves the exact radiative transfer equation and accurate spectral radiative properties have bee considered to account for strong spectral correlation effects in gases. Thus, regarding the description of the radiative energy transfer, the level of physical fidelity is in line with DNS. An instantaneous snapshot of the solution fields is shown in figure 1. The study focuses here on radiation effects on higher-order statistical moments such as turbulent transport heat flux and enthalpy root-mean-square (RMS). The modification of their respective budget equations is also investigated.

After a detailed description of the studied problem in section 2, effects of radiation are analyzed in a first channel flow configuration in section 3.1. Then, changes of radiation effects with wall temperature difference and bulk Reynolds number are reported in section 3.2 where a new turbulent scaling is proposed. Finally, the results related to the turbulent Prandtl number are given in section 3.3 and a model based on the proposed scaling is derived.

\section{Problem description}

In order to study accurately the effects of radiation on the structure of turbulent boundary layers, direct numerical simulations of a planar channel flow coupled with a reciprocal Monte-Carlo method for radiation calculations have been considered. The set of governing equations in the fluid is given by

$$
\begin{gathered}
\frac{\partial \rho}{\partial t}+\frac{\partial\left(\rho u_{i}\right)}{\partial x_{i}}=0 \\
\frac{\partial\left(\rho u_{i}\right)}{\partial t}+\frac{\partial\left(\rho u_{i} u_{j}\right)}{\partial x_{j}}=-\frac{\partial p}{\partial x_{i}}+\frac{\partial \tau_{i j}}{\partial x_{j}}+S_{i}, \\
\frac{\partial(\rho \mathrm{h})}{\partial t}+\frac{\partial\left(\rho u_{j} \mathrm{~h}\right)}{\partial x_{j}}=\frac{\partial p}{\partial t}-\frac{\partial q_{j}^{c d}}{\partial x_{j}}+P^{R} \\
p=\rho r T
\end{gathered}
$$

where $\rho, u_{i}, \mathrm{~h}, p$ and $T$ are the fluid mass density, velocity components, enthalpy per mass units, pressure and temperature, respectively. $\mathrm{h}$ is expressed from the mixture thermal capacity at constant pressure $c_{p}: \mathrm{h}=\Delta \mathrm{h}_{0}+\int_{T_{0}}^{T} c_{p}\left(T^{\prime}\right) d T^{\prime}$, where $T_{0}$ is a reference temperature and $\Delta \mathrm{h}_{0}$ the corresponding standard formation enthalpy. The viscous shear stress tensor $\tau_{i j}$ and the conductive flux vector $q_{i}^{c d}$ are

$$
\begin{gathered}
\tau_{i j}=\mu\left(\frac{\partial u_{i}}{\partial x_{j}}+\frac{\partial u_{j}}{\partial x_{i}}\right)-\frac{2}{3} \mu\left(\frac{\partial u_{k}}{\partial x_{k}}\right) \delta_{i j}, \\
q_{i}^{c d}=-\lambda \frac{\partial T}{\partial x_{i}}
\end{gathered}
$$

where $\mu$ is the dynamic viscosity, function of temperature computed like the mixture thermal capacity $c_{p}$ by the CHEMKIN package (Kee et al. 1986, 1989). The thermal conductivity $\lambda$ is computed from the Prandtl number $\operatorname{Pr}=0.71 . P^{R}$ is the radiative power per unit volume. $S_{i}$ is a uniform forcing source term which acts as a pressure gradient term and drives the channel flow to obtain the desired bulk Reynolds number $\mathrm{Re}_{b}$.

The set of governing equations is solved with the finite-volume solver YALES2 (Moureau et al. 2011a,b) under a low Mach-number approximation. As detailed in Zhang et al. 


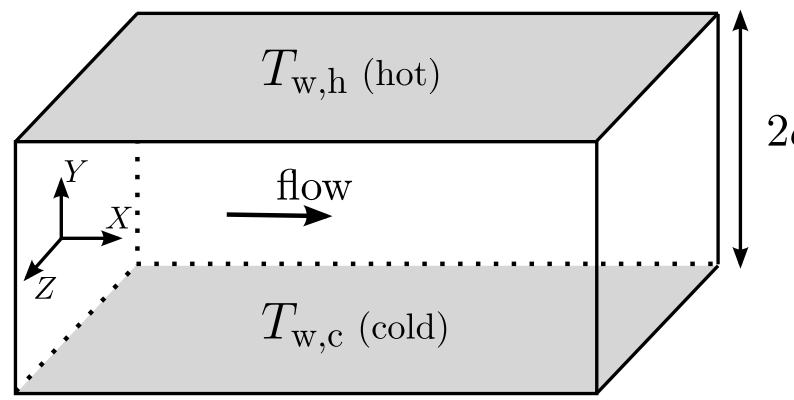

Figure 2. Computational domain of channel flow of half-width $\delta=0.1 \mathrm{~m}$. The lower wall (resp. upper wall) is at temperature $T_{w, c}\left(\operatorname{resp} . T_{w, h} ; T_{w, h} \geqslant T_{w, c}\right)$.

$\begin{array}{ccccc}\text { Case } & \mathrm{Re}_{b} & T_{\mathrm{w}, \mathrm{c}}[\mathrm{K}] & T_{\mathrm{w}, \mathrm{h}}[\mathrm{K}] & p[\mathrm{~atm}] \\ \mathrm{A} & 5850 & 950 & 1150 & 40.0 \\ \mathrm{~B} & 5850 & 950 & 2050 & 40.0 \\ \mathrm{C} & 11750 & 950 & 1150 & 40.0\end{array}$

TABLE 1. Channel flow parameters: Bulk Reynolds number $\mathrm{Re}_{b}$, wall temperatures and pressure.

(2013a), the numerical setup is composed of a centered fourth-order spatial discretization and a fourth-order time integration. The exact radiative transfer equation is solved with a Monte-Carlo method. The computation of the radiative power is here handled by an Optimized Emission-based Reciprocity Monte-carlo method (OERM) (Zhang et al. $2013 a$ ) where the statistical error on the radiative power is set to remain below $3 \%$ of the radiative power maximum value. In the radiative transfer solver, each grid cell is assumed isothermal. Spectral correlation effects in gases are very strong and it is outlined that the gray gas assumption is a myth, see Edwards (1976). In principle, the most accurate approach is a line-by-line model associated with a high-resolution and accurate spectroscopy database. Even with a Monte-Carlo method, such an approach cannot be carried out for unsteady coupled 3D simulations. To our knowledge, the today most accurate practical approach is the CK method based on accurate parameters directly issued from a high-resolution database (Taine \& Soufiani 1999). In our case, the high resolution computations account for special effects of pressure on the spectral line wings that are not anymore lorentzian, and of line couplings (Perrin \& Hartmann 1989). At high pressure, the weak absorption limit of the CK approach is accurate and also account for the aforementioned effect. This latter spectral description of gases properties has been retained here.

The studied configuration, a fully developed turbulent channel flow with two isothermal walls, is shown in figure 2. The computational domain is $2 \pi \delta \times 2 \delta \times \pi \delta$ where $\delta=$ $0.1 \mathrm{~m}$. Periodic boundary conditions are applied along $\mathrm{X}$ and $\mathrm{Z}$ directions. For radiation simulations, if a shot exits the domain, for instance, at the point $\left(L_{X}, Y, Z\right)$, it will then enter at the point $(0, Y, Z)$ with the same propagation direction. The medium is a non-reacting $\mathrm{CO}_{2}-\mathrm{H}_{2} \mathrm{O}-\mathrm{N}_{2}$ gas mixture characterized by the respective molar fractions 0.116-0.155-0.729.

Three computational cases from Zhang et al. (2013a) (cases C1, C3 and C4), called here $\mathrm{A}, \mathrm{B}$ and $\mathrm{C}$, are defined in table 1 by a set of bulk Reynolds number, pressure and wall temperatures $\left(T_{\mathrm{w}, \mathrm{c}}\right.$ and $\left.T_{\mathrm{w}, \mathrm{h}}\right)$. Only high pressure cases are considered to enhance 
radiative energy transfer. The three investigated cases $\mathrm{A}, \mathrm{B}$ and $\mathrm{C}$ are characterized by a global medium Hotell's transmissivity at $1000 \mathrm{~K}$ of 0.271 , which indicates a large optical thickness of the medium. In optically thinner cases such as atmospheric conditions, the radiative effects on the mean temperature profile has been observed to be similar to the ones at high pressure but smaller in magnitude (Zhang et al. 2013a). In case C, a wall temperature over $2000 \mathrm{~K}$ does not correspond to any practical combustion systems but the purpose is to have high temperature burnt gases away from the cold wall as found in combustors with large heat loads. The bulk Reynolds number $\mathrm{Re}_{b}$ is defined by

$$
\operatorname{Re}_{b}=\frac{\rho_{b} u_{b} \delta}{\mu_{b}} \quad \text { with } \quad \rho_{b}=\frac{\int_{-\delta}^{\delta} \bar{\rho} d Y}{2 \delta}, \quad u_{b}=\frac{\int_{-\delta}^{\delta} \overline{\rho u} d Y}{\int_{-\delta}^{\delta} \bar{\rho} d Y}, \quad \mu_{b}=\mu\left(T_{b}\right)
$$

where $T_{b}$ is the bulk temperature defined from the bulk enthalpy:

$$
\mathrm{h}\left(T_{b}\right)=\frac{\int_{-\delta}^{\delta} \overline{\rho u \mathrm{~h}} d Y}{\int_{-\delta}^{\delta} \overline{\rho u} d Y}
$$

Discretization details for each case are given in table 2. Cell sizes $\Delta X$ and $\Delta Z$ in the streamwise and spanwise directions are uniform, while the cell length $\Delta Y$ is refined close to the walls. Non-dimensional cell sizes in table 2 are expressed in wall units from the wall mean density, viscosity and the friction velocity similarly to equation (3.2). When radiation is considered, these cases are referred as A_R, B_R and C_R, respectively and the emissivity $\varepsilon$ of the opaque walls is set to 0.8 . Cases with radiation A_R, B_R and C_R correspond to cases C1R1, C3R1 and C4R1 from Zhang et al. (2013a), respectively. These coupled direct numerical simulations with radiative energy transfer are five to ten times more expensive computationally than DNS without radiation. Because of the large computational cost of the radiation solver, the grid used for the radiation model is three times coarser in $\mathrm{X}$ direction and twice in $\mathrm{Y}$ and $\mathrm{Z}$ directions than the corresponding grid of the flow field. For the same reason, the radiative source term in the energy equation is updated every three time steps of the fluid solver. Such considerations can have an impact on the accuracy of the simulated turbulence-radiation interaction. Since the numerically well-resolved larger turbulent scales are mainly involved in this interaction, the impact of the present grid coarsening and update frequency of the radiative power is expected to be small. Besides, the variations of radiative power are located in the vicinity of the walls where the grid (the coarse one included) is refined. In order to carefully assess this effect, the radiative power was computed for a couple of instantaneous solutions on the fine grid with a stricter threshold on the statistical error to provide reference solutions. On instantaneous fields, small differences in the radiative power with the coarse grid results and default threshold are noticeable. However, as proper comparisons of turbulent fields requires statistical averages, the solutions have been averaged in homogeneous directions $\mathrm{X}$ and $\mathrm{Z}$. It was finally verified that the single-point statistical first and second-order moments that involve the radiative power and that are here presented are not sensitive to the grid coarsening.

Without additional care, the Reynolds numbers differ between simulations with or without radiation. The source term in the momentum equation is adapted in order to keep the same bulk Reynolds number in both configurations. The friction Reynolds number based on the friction velocity in the channel boundary layers is nonetheless slightly different with or without radiation. Values have been reported in Zhang et al. (2013a). However, at least one characteristic of the flow, here chosen as the bulk Reynolds number, is consistent across simulations. 


$\begin{array}{ccccc}\text { Case } & n_{X} \times n_{Y} \times n_{Z} & \begin{array}{c}\Delta X^{+} \\ \text {hot/cold }\end{array} & \Delta Y^{+} & \begin{array}{c}\Delta Z^{+} \\ \text {hot/cold }\end{array} \\ \text { A } & 110 \times 135 \times 110 & 16.9 / 21.3 & {[0.8-8.0]} & 8.4 / 10.6 \\ \text { B } & 160 \times 163 \times 160 & 8.9 / 22.4 & {[0.8-8.0]} & 4.4 / 11.2 \\ \text { C } & 200 \times 230 \times 200 & 17.3 / 21.8 & {[0.8-8.0]} & 8.6 / 10.8\end{array}$

TABLE 2. Discretization of the simulated cases: $n_{X}$, respectively $n_{Y}$ and $n_{Z}$, is the number of points in the $X$ direction, respectively $Y$ and $Z$ direction. $\Delta X^{+}$and $\Delta Z^{+}$are given at the cold and hot sides for cases $\mathrm{A}, \mathrm{B}$ and $\mathrm{C}$ without radiation.

\section{Results}

\subsection{Results for reference cases $A$ and $A_{-} R$}

Results are first presented for cases A and A_R. The effects of radiation on the boundary layer structure are analyzed in terms of the effects on the mean temperature field, on the enthalpy fluctuations and on the turbulent transport heat flux. Then, influence of temperature fluctuations on the radiative power is studied. In the following, $\bar{\phi}$ and $\widetilde{\phi}$ denote Reynolds and Favre averages for any variable $\phi$, respectively, while $\phi^{\prime}$ and $\phi^{\prime \prime}$ denote their respective fluctuating parts.

\subsubsection{Mean temperature field}

Owing to the small variation of mass density in the present conditions, mean velocity profiles (not presented here) are not affected by radiation when comparing cases A and A_R that are both characterized by a small wall temperature difference. However, the mean temperature profile, shown in figure 3 (a), is significantly modified by radiation over the whole domain. The mean temperature in wall units $\bar{T}^{+}$is defined as,

$$
\bar{T}^{+}=\frac{\left|\bar{T}-T_{w}\right|}{T_{\tau}} \quad \text { with } \quad T_{\tau}=\frac{\left|\overline{q_{w}^{c d}}\right|}{\overline{\rho_{w}} \overline{c_{p_{w}}} u_{\tau}}, u_{\tau}=\left(\frac{\overline{\tau_{w}}}{\overline{\rho_{w}}}\right)^{1 / 2},
$$

where $\overline{q_{w}^{c d}}, \overline{\rho_{w}}, \overline{c_{p_{w}}}$ and $\overline{\tau_{w}}$ are the mean conductive heat flux, mass density, thermal capacity and shear stress at the wall, respectively. The $\bar{T}^{+}$profiles of cases A and A_R are plotted in figure 3 (b) as functions of the normalized wall distance $y^{+}$that is given by,

$$
y^{+}=\frac{\overline{\rho_{w}} y u_{\tau}}{\overline{\mu_{w}}}
$$

where $\overline{\mu_{w}}$ is the mean dynamic viscosity at the wall. The obtained temperature wall law for case A_R strongly deviates from that of case A, showing a significant effect of radiation on the thermal boundary layer structure in the considered conditions. A detailed analysis of such radiation effects on the mean temperature field in different channel flow conditions has been realized (Zhang et al. 2013a). Opposite effects of radiation on the wall conductive heat flux and on the temperature wall law have been observed and they have been explained by the antagonist behaviors of gas-gas and gas-wall radiative contributions.

\subsubsection{Fluctuations of enthalpy}

Profiles of the enthalpy root-mean-square, $\mathrm{h}_{\mathrm{rms}}=\left(\widetilde{\mathrm{h}^{\prime \prime} \mathrm{h}^{\prime \prime}}\right)^{1 / 2}$, in cases A and A_R are shown in figure 4 (a), where $\mathrm{h}_{\mathrm{rms}}$ is scaled by the arithmetic mean of the wall temperatures 

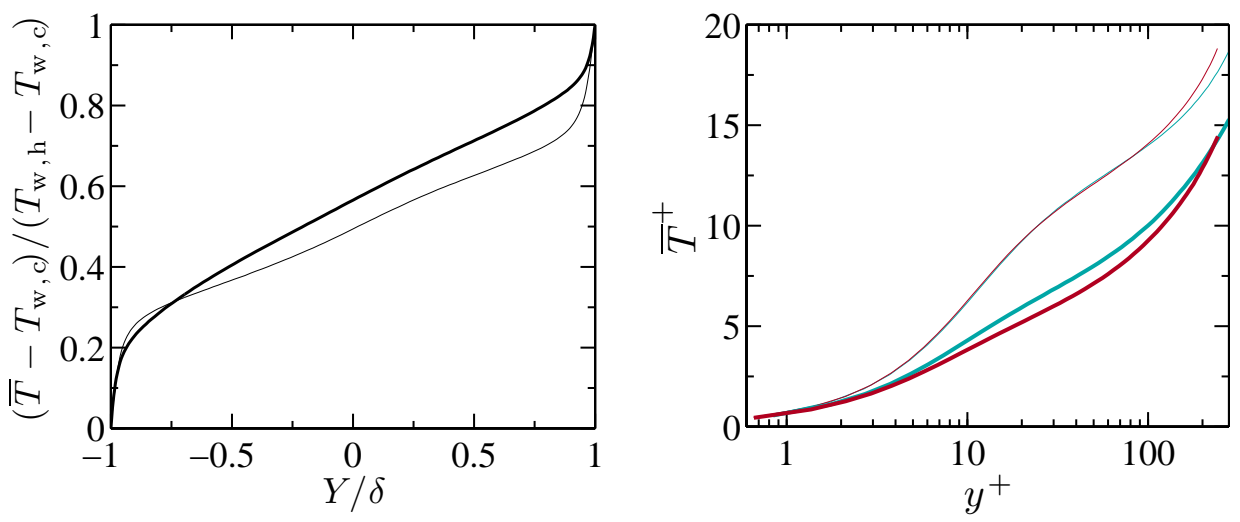

(a)

Figure 3. Profiles of mean temperature scaled by wall temperatures (a) and in wall units (b) on the cold side (blue color) and hot side (red color) in cases A (thin line) and A_R (thick line).

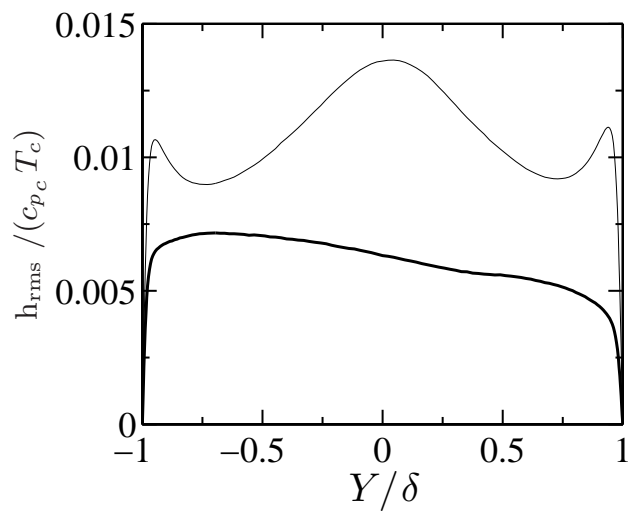

(a)

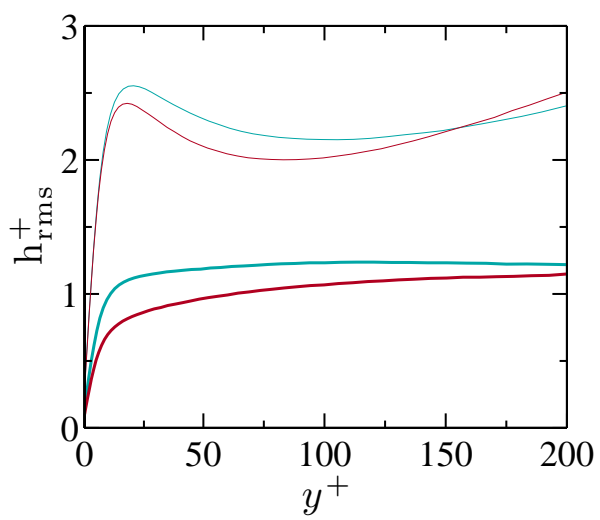

(b)

FIGURE 4. Profiles of enthalpy root-mean-square scaled by center temperature $T_{c}$ and thermal capacity $c_{p_{c}}$ (a) and in wall units (b) on the cold side (blue color) and hot side (red color) in cases A (thin line) and A_R (thick line).

$T_{c}=\left(T_{\mathrm{w}, \mathrm{c}}+T_{\mathrm{w}, \mathrm{h}}\right) / 2$ and the arithmetic mean thermal capacity $c_{p, c}$, defined as the mean between the thermal capacities at the walls. In case A, without radiation, peaks of variance are located in the near wall regions as expected from standard boundary layer theory where production of turbulent fluctuations is maximal within the buffer layer. Because of the specific configuration where wall temperatures are different, a larger peak in $\mathrm{h}_{\mathrm{rms}}$ appears in the core of the channel where, as explained by Debusschere \& Rutland (2004), fluid pockets of high and low temperature converge from the hot and cold walls, respectively. In case A_R where radiation is accounted for, a significant reduction in enthalpy fluctuations is observed in the near wall region, especially on the hot side, and the central peak vanishes.

One effect of radiation on the absolute value of $h_{r m s}$ is the change in the mean wall conductive heat flux due to the modified mean temperature profile. Most of this effect can be filtered out by rescaling profiles with the friction temperature. The obtained wall-scaled profiles of enthalpy rms against $y^{+}$are presented in figure 4 (b), where the 




(a)

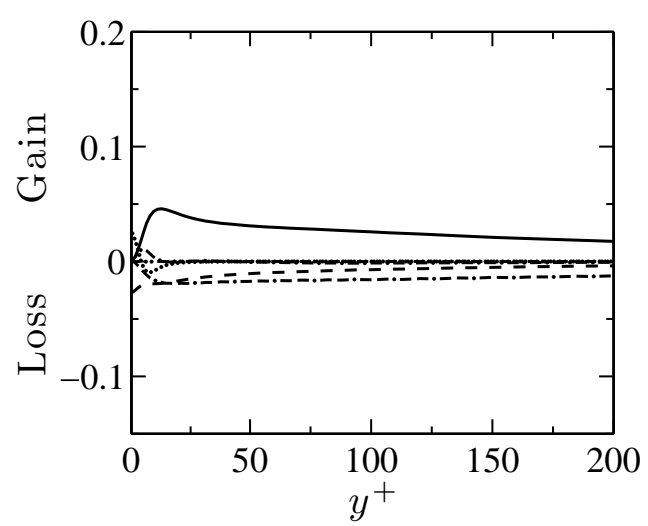

(b)

Figure 5. Budget of enthalpy variance (cold side only) in cases A (a) and A_R (b): Production (plain line); Molecular dissipation (dashed line); Radiative dissipation (dashed-dashed-dotted line); Turbulent diffusion (dashed-dotted line); Molecular diffusion (dotted line); Density-enthalpy correlation term (dashed-dotted-dotted line).

non-dimensional enthalpy $\mathrm{rms}_{\mathrm{rms}}^{+}$is defined as

$$
\mathrm{h}_{\mathrm{rms}}^{+}=\frac{\mathrm{h}_{\mathrm{rms}}}{\overline{c_{p_{w}}} T_{\tau}} \text {. }
$$

In spite of the wall scaling formulation, a large difference between results of cases $\mathrm{A}$ and A_R remains, indicating that the strong effect of radiation on fluctuations of enthalpy and temperature is a real modification of the boundary layer structure.

This point is further investigated by analyzing the balance of the enthalpy variance transport equation (see Appendix A) which, in the studied configuration, is

$$
\underbrace{-\frac{\partial}{\partial y}\left(\overline{q_{y}^{c d^{\prime}} \mathrm{h}^{\prime}}\right)}_{\text {I }} \underbrace{-\frac{1}{2} \frac{\partial}{\partial y}\left(\bar{\rho} \widetilde{v^{\prime \prime} \mathrm{h}^{\prime \prime} \mathrm{h}^{\prime \prime}}\right)}_{\text {II }} \underbrace{-\widetilde{\rho v^{\prime \prime} \mathrm{h}^{\prime \prime} \frac{\partial \tilde{\mathrm{h}}}{\partial y}}}_{\text {III }}+\underbrace{\overline{q_{i}^{c d^{\prime}} \frac{\partial \mathrm{h}^{\prime}}{\partial x_{i}}}}_{\text {IV }}+\underbrace{\overline{\mathrm{h}^{\prime \prime} P^{R^{\prime}}}}_{\mathrm{V}}+\underbrace{\overline{\mathrm{h}^{\prime \prime}} \frac{\partial}{\partial y}\left(\bar{\rho} \widetilde{v^{\prime \prime} \mathrm{h}^{\prime \prime}}\right)}_{\text {VI }}=0
$$

where the terms on the left hand side are molecular diffusion (I), turbulent diffusion (II), production (III), molecular dissipation (IV), correlation between enthalpy and radiative power fluctuations $(\mathrm{V})$ and a term $(\mathrm{VI})$ proportional to $\overline{\mathrm{h}^{\prime \prime}}=-\frac{\overline{\rho^{\prime} h^{\prime}}}{\bar{\rho}}$ related to enthalpydensity correlation. For any quantities $\psi$ and $\phi$, cross-correlations between Favre and Reynolds fluctuations have the following properties: $\overline{\phi^{\prime} \psi^{\prime \prime}}=\overline{\phi^{\prime} \psi^{\prime}}$ and $\widetilde{\phi^{\prime} \psi^{\prime \prime}}=\widetilde{\phi^{\prime \prime} \psi^{\prime \prime}}$. Therefore, the correlation term between enthalpy and radiative power fluctuations can also be written as $\overline{\mathrm{h}^{\prime} P^{R^{\prime}}}$.

The different terms in equation (3.4) are scaled in wall units by ${\overline{q_{w}^{c d}}}^{2} / \overline{\mu_{w}}$ and compared in figure 5 (a) and (b) for cases A and A_R (only the results on the cold side are shown since they are similar on the hot side). On the one hand, in case A, production and molecular dissipation terms are dominant and decrease away from the wall as expected in such standard conditions. On the other hand, in case A_R, a third dominant term appears in the balance of enthalpy variance in addition to the latter two, that is the enthalpyradiative-power correlation. Since this term appears as a negative contribution to the budget, it will be referred as radiative dissipation in the following. Hence, equilibrium between production and molecular dissipation away from the buffer layer for $y^{+}>30$ is replaced by a balance of production with molecular and radiative dissipations in the case with radiation. In the studied case, this equilibrium takes place sooner for $y^{+}>$ 


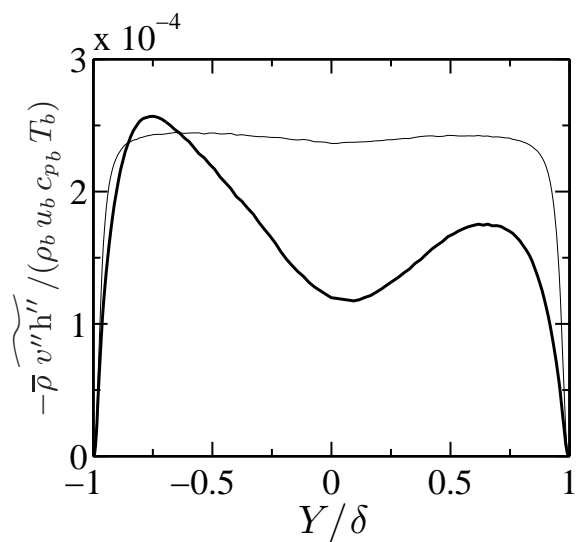

(a)

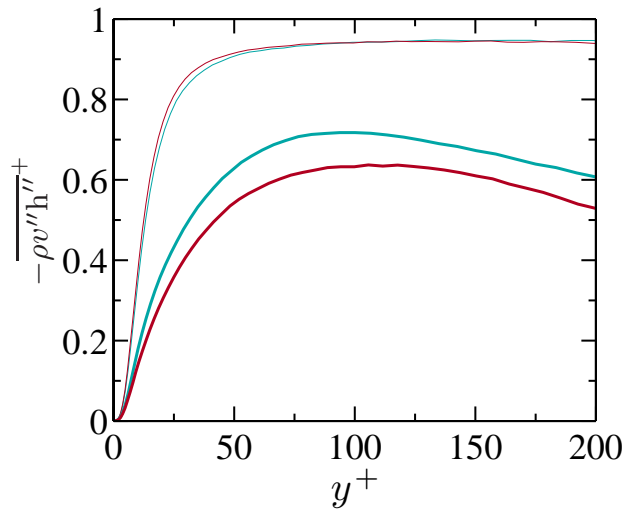

(b)

Figure 6. Profiles of wall-normal turbulent heat flux scaled by bulk variables (a) and in wall-units (b) on the cold side (blue color) and hot side (red color) in cases A (thin line) and A_R (thick line).



Figure 7. Profiles of correlation between enthalpy and wall-normal velocity fluctuations in cases A (thin line) and A_R (thick line).

20 and molecular dissipation remains weaker than radiative dissipation for $y^{+}>50$ approximatively.

For both cases A and A_R, the term related to $\overline{\mathrm{h}^{\prime \prime}}$, mean of mass-weighted fluctuating enthalpy, is negligible because of the small density variations. Regarding the scaled production, it is reduced in case A_R because of the modification by radiation of the mean temperature field and of the turbulent heat flux (shown later). This decrease in production and the presence of an additional radiation-related dissipative term in the budget of enthalpy variance explain the smaller level of enthalpy fluctuations shown in figure 4 for the case with radiation. 


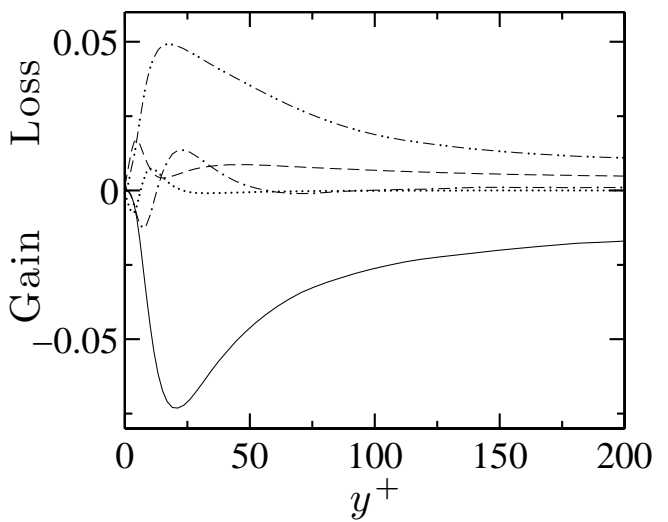

(a)

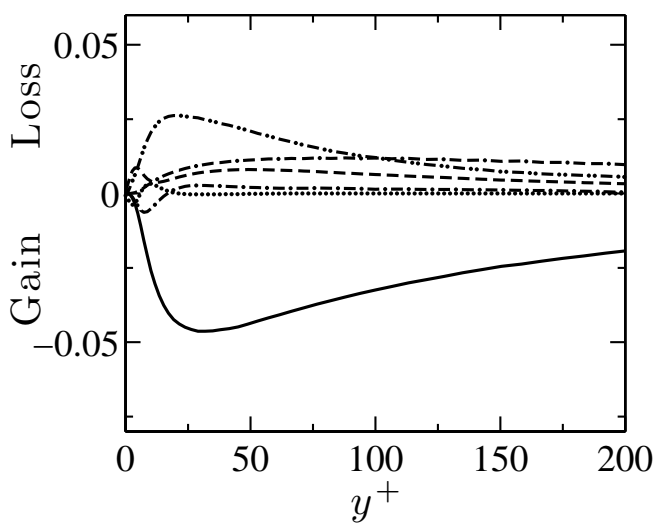

(b)

FiguRE 8. Budget of wall-normal turbulent heat flux (cold side only) in cases A (a) and A_R (b): Production (plain line); Molecular diffusion (dotted line); Turbulent diffusion (dashed-dotted line); Enthalpy-pressure-gradient correlation (dashed-dotted-dotted line); Molecular dissipation (dashed line); Velocity-Radiative power correlation term (dashed-dashed-dotted line).

\subsubsection{Wall-normal turbulent heat flux}

Profiles of wall-normal turbulent heat flux $\widetilde{\rho} \widetilde{v^{\prime \prime} \mathrm{h}^{\prime \prime}}$ in cases A and A_R are presented in figure 6 , where the wall-scaled turbulent heat flux is defined as

$$
\overline{\rho v^{\prime \prime} \mathrm{h}^{\prime \prime}}+=\frac{\widetilde{\rho} v^{\prime \prime} \mathrm{h}^{\prime \prime}}{\bar{\rho}_{w} u_{\tau} \overline{c_{p_{w}}} T_{\tau}}=\frac{\widetilde{\rho} v^{\prime \prime} \mathrm{h}^{\prime \prime}}{\left|\overline{q_{w}^{c d}}\right|} .
$$

The scaled wall-normal turbulent heat flux decreases when radiation is accounted for. The aforementioned reduction in enthalpy fluctuations due to radiation is a first explanation why the turbulent transfer is less efficient. This effect can be filtered out by looking at the correlation coefficient between enthalpy and wall-normal velocity fluctuations shown in figure 7. The resulting profile for case A_R is symmetrical, which shows that the asymmetry observed in figure 6 (a) for the case with radiation comes from the asymmetry of $h_{\text {rms }}$ between the hot and cold walls (figure 4). The correlation between enthalpy and wall-normal velocity fluctuations is nonetheless stronger when radiation is accounted for. Other sources of disagreement between the two cases A and A_R than just the change in enthalpy rms are therefore at work.

One of these sources is the requirement for the turbulent heat flux to fulfill the mean balance equation of energy that is given by,

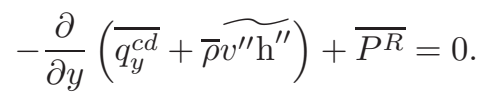

Introducing the radiative flux vector $q_{i}^{R}$, the mean energy balance equation becomes a constant sum of energy fluxes,

$$
\overline{q_{y}^{c d}}+\widetilde{\rho v^{\prime \prime} \mathrm{h}^{\prime \prime}}+\overline{q_{y}^{R}}=\overline{q_{w}^{c d}}+\overline{q_{w}^{R}},
$$

where $\overline{q_{w}^{c d}}$ and $\overline{q_{w}^{R}}$ are the wall conductive heat flux and wall radiative flux, respectively. Hence, the turbulent heat flux is constrained by conductive and radiative fluxes, establishing a strong two-way coupling of the radiative power field with the mean and the fluctuations of the temperature field. 
The balance equation of the turbulent heat flux $\widetilde{\rho v^{\prime \prime} h^{\prime \prime}}$ (see Appendix A) is

$$
\begin{aligned}
& \underbrace{\frac{\partial}{\partial y}\left(\overline{\tau_{22}^{\prime} \mathrm{h}^{\prime}}-\overline{q_{y}^{c d^{\prime}} v^{\prime}}\right)}_{\mathrm{I}} \underbrace{-\frac{\partial}{\partial y}\left(\bar{\rho} \widehat{v^{\prime \prime} v^{\prime \prime} \mathrm{h}^{\prime \prime}}\right)}_{\mathrm{II}} \underbrace{-\bar{\rho} \widehat{v^{\prime \prime} v^{\prime \prime} \frac{\partial \mathrm{h}}{\partial y}}}_{\mathrm{III}} \underbrace{\left.-\overline{\left(\tau_{2 i}^{\prime} \frac{\partial \mathrm{h}^{\prime}}{\partial x_{i}}\right.}-\overline{q_{i}^{c d^{\prime}} \frac{\partial v^{\prime}}{\partial x_{i}}}\right)}_{\mathrm{IV}} \\
& +\underbrace{\overline{v^{\prime \prime} P^{R^{\prime}}}}_{\text {V }} \underbrace{-\overline{\mathrm{h}^{\prime} \frac{\partial p^{\prime}}{\partial y}}}_{\text {VI }}+\underbrace{\left(\overline{\mathrm{h}^{\prime \prime}} \frac{\partial}{\partial y}\left(\bar{\rho} \widetilde{v^{\prime \prime} v^{\prime \prime}}\right)+\overline{v^{\prime \prime}} \frac{\partial}{\partial y}\left(\bar{\rho} \widetilde{v^{\prime \prime} \mathrm{h}^{\prime \prime}}\right)\right)}_{\text {VII }}=0 .
\end{aligned}
$$

The seven terms on the left hand side are molecular diffusion (I), turbulent diffusion (II), production (III), molecular dissipation (IV), correlation between wall-normal velocity and radiative power fluctuations (V), enthalpy-pressure-gradient correlation (VI: EPG) and a term (VII) related to the average of Favre fluctuations of enthalpy and velocity. These terms, except for the last one which is again negligible, are shown for cases A and A_R in figure 8 where they are scaled by $\left|\overline{q_{w}^{c d}}\right| \overline{\tau_{w}} / \overline{\mu_{w}}$. In case A, the predominant terms are the production and the EPG terms. Away from the wall, molecular dissipation accounts for the remaining balance. The enthalpy-pressure-gradient correlation can be split into two terms, a pressure-enthalpy-gradient correlation and a pressure-diffusion term:

$$
\overline{-\mathrm{h}^{\prime} \frac{\partial p^{\prime}}{\partial y}}=\overline{p^{\prime} \frac{\partial \mathrm{h}^{\prime}}{\partial y}}-\frac{\partial \overline{p^{\prime} \mathrm{h}^{\prime}}}{\partial y}
$$

As shown by Kasagi et al. (1992), these two terms are of the same order of magnitude with opposite signs (not presented here). When radiation is taken into account, most of the terms are reduced in magnitude and broader. For $y^{+}>25$, the balance is split into production, the EPG term, the wall-normal velocity-radiative power correlation term and molecular dissipation which has the smallest contribution among these four terms. The EPG term is the largest loss term for $y^{+}<100$ until the velocity-radiative power correlation term takes over.

At large Reynolds number, the molecular dissipation in the balance equation of the turbulent heat flux becomes negligible away from the wall (Pope 2000). Extrapolating the present results to flows with a larger Reynolds number, two different types of behaviors can be expected away from the wall: i) When radiation is negligible or moderate, production of turbulent heat flux is equilibrated with the EPG contribution only; ii) When radiation is intense, a second region appears further away from the wall where production is equilibrated with the wall-normal velocity-radiative power correlation term only. For stronger and stronger radiative effects, the area where the radiative term becomes dominant gets closer and closer to the wall.

\subsubsection{Radiative power field}

The mean profile of the radiative power is shown in figure 9 (a). It is positive (resp. negative) in the very near wall region on the cold side (resp. hot side). Further away from the wall, the radiative power changes sign twice around $y^{+}=20$ and 100 on both sides. This shape of the mean radiative power has been explained by the different behaviors of gas-gas and gas-wall contributions to radiation (Zhang et al. 2013a).

One of the most studied aspects of turbulence-radiation interaction is the effect of turbulent fluctuations on the mean radiative power (Coelho 2012). Indeed, the mean 




(a)

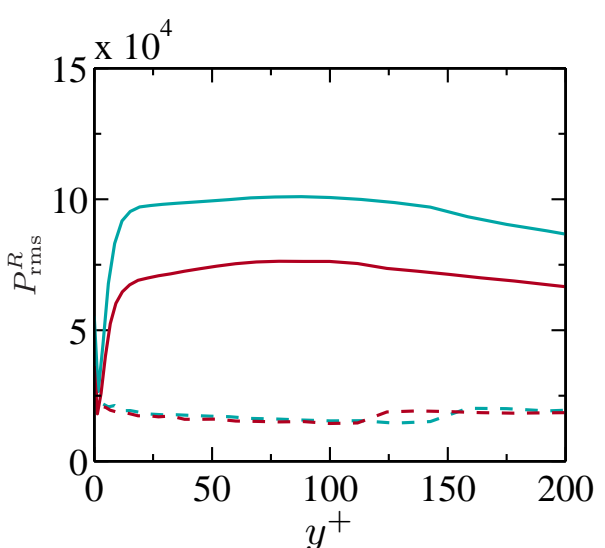

(b)

FiguRE 9. Profiles of mean radiative power $\overline{P^{R}}$ (a) and root-mean-square $P^{R}$ rms (b) in J.m ${ }^{-3}$ in case A_R on the cold side (blue color) and hot side (red color). (a) Circles : radiative power computed from the mean temperature field. (b) Plain lines: physical rms; Dashed lines: stochastic error.

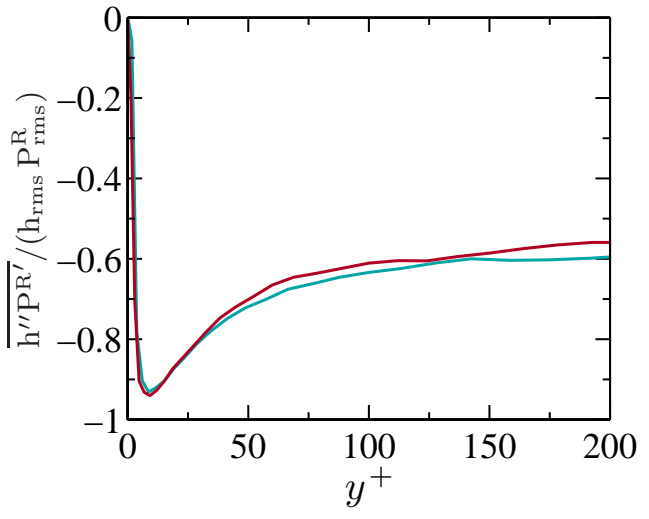

(a)

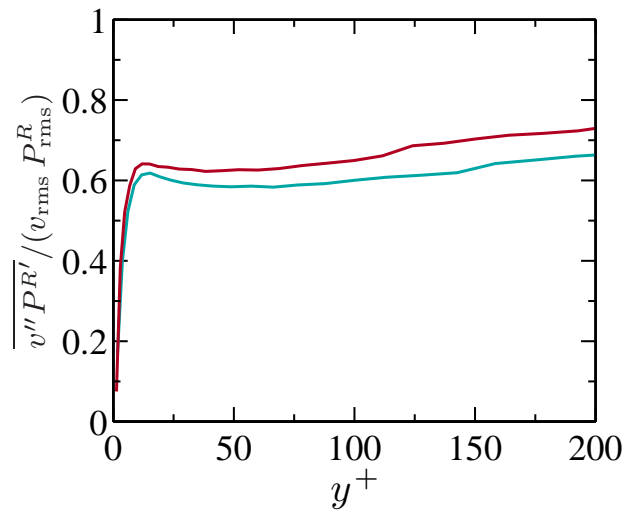

(b)

Figure 10. Correlation coefficients between radiative power and enthalpy (a) and between radiative power and wall-normal velocity (b) in case A_R on the cold side (blue color) and hot side (red color).

radiative power is given by

$$
\overline{P^{R}}=\overline{P_{a}^{R}}-\overline{P_{e}^{R}}=\int_{0}^{+\infty}(\underbrace{\int_{4 \pi} \overline{\kappa_{\nu} I_{\nu}} \mathrm{d} \Omega}_{\text {Absorption }}-\underbrace{4 \pi \overline{\kappa_{\nu} I_{\nu}^{\circ}(T)}}_{\text {Emission }}) \mathrm{d} \nu,
$$

where the spectral intensity $I_{\nu}$, the equilibrium spectral intensity $I_{\nu}^{\circ}$ and the spectral absorption coefficient $\kappa_{\nu}$ are integrated over frequency $\nu$ and solid angle $\Omega$ ranges. While $I_{\nu}^{\circ}$ depends on the local temperature and $\kappa_{\nu}$ on the local state (composition, pressure and temperature), the spectral intensity $I_{\nu}$ at a given point is determined by the whole three-dimensional and instantaneous fields of temperature, pressure and composition. This latter property of the spectral intensity has led to numerous studies on the effects of turbulents fluctuations on the mean radiative power (Coelho 2007, 2012) since the mean radiative power cannot be computed from the mean fields of temperature, pressure 
and composition as they do not correspond to any instantaneous realization of the flow. In the present study, channel flows with homogenous composition and thermodynamic pressure are considered. Therefore, only the temperature field determines the radiative fields and $\overline{P^{R}}$ cannot be computed in the general case from the three-dimensional field of mean temperature $\{\bar{T}\}$, i.e.

$$
\overline{P^{R}(\{T\})} \neq P^{R}(\{\bar{T}\}) .
$$

To investigate this effect in the present case, the radiative power computed from the mean temperature field is also shown in figure 9 (a). No difference appears with the exact profile on both sides, where emission is dominant in the near-wall region of the hot wall, while absorption is dominant close to the cold wall. Consequently, turbulent fluctuations of temperature are not intense enough in the present boundary layer case to perturb the mean emitted and absorbed radiative power. This was also reported by Gupta et al. (2009) for non-reactive large-eddy simulation of channel flow. Due to the complexity of the absorption contribution, most studies in Turbulence-Radiation Interaction (TRI) focus on the effect of turbulent fluctuations on the emitted radiative power. Introducing the Planck mean absorption coefficient $\kappa_{P}$ and neglecting high order correlation, the mean emitted radiative power is (Coelho 2007):

$$
\overline{P_{e}^{R}}=\overline{4 \pi \int_{0}^{+\infty} \kappa_{\nu} I_{\nu}^{0}(T) \mathrm{d} \nu}=\overline{4 \sigma \kappa_{P} T^{4}}=\underbrace{4 \sigma \bar{T}^{4} \overline{\kappa_{P}}}_{=P_{e}^{R}(\bar{T})}\left(1+6 \frac{\overline{T^{2}}}{\bar{T}^{2}}+4 \frac{\overline{\kappa_{P}^{\prime} T^{\prime}}}{\overline{\kappa_{P}} \bar{T}}+\cdots\right)
$$

where $\sigma$ is the Stefan-Boltzmann constant. In the present study with homogenous composition and pressure, the Planck mean absorption coefficient only depends on temperature. As fluctuations of temperature in turbulent boundary layers remain moderate (see Appendix $\mathrm{B}), \kappa_{P}(T) \approx \kappa_{P}(\bar{T})+\alpha T^{\prime}$ can be linearized around the mean temperature $\bar{T}$, and the relative variation in the Planck mean absorption coefficient is given by

$$
\frac{\kappa_{P}(T)-\kappa_{P}(\bar{T})}{\kappa_{P}(\bar{T})}=\beta \frac{T^{\prime}}{\bar{T}}
$$

with $\beta=\alpha \bar{T} / \kappa_{P}(\bar{T})$. Finally, the effect of temperature fluctuations on the mean emitted power, quantified as the relative difference between $\overline{P_{e}^{R}}$ and $P_{e}^{R}(\bar{T})$, is

$$
\frac{\overline{P_{e}^{R}}-P_{e}^{R}(\bar{T})}{P_{e}^{R}(\bar{T})}=(\underbrace{6+4 \beta}_{\gamma}) \frac{\overline{T^{\prime 2}}}{\bar{T}^{2}} \text {. }
$$

For fluctuating level of temperature $T^{\mathrm{rms}} / \bar{T}$ larger than $1 / \gamma, \mathrm{X} \%$ of temperature fluctuations results in TRI effects on the mean emitted radiative power larger than $\mathrm{X} \%$, and reciprocally. Dependency of the Planck mean absorption coefficient with temperature for $\mathrm{H}_{2} \mathrm{O}$ and $\mathrm{CO}_{2}$ are given by Rivière \& Soufiani (2012), which shows that the introduced proportionality coefficient $\beta$ varies between -1 (resp. 0.5 ) at $500 \mathrm{~K}$ and -2.3 (resp. -2.5) at $2500 \mathrm{~K}$ for $\mathrm{H}_{2} \mathrm{O}$ (resp. $\mathrm{CO}_{2}$ ). Hence, for various composition of exhaust gases, $\beta$ ranges between -2.5 and 0.5 , yielding $|\gamma|$ within the range $4-8$. Consequently, the threshold between amplification and diminution of TRI effects on $\overline{P_{e}^{R}}$ corresponds to approximatively $12-25 \%$ of fluctuations in temperature. In the present case, maximum temperature fluctuations reach roughly $1 \%$ in cases $\mathrm{A}$ and $\mathrm{C}$, and $5 \%$ in case $\mathrm{B}$. When radiation is considered, these fluctuations are even lower as explained previously. With such levels of turbulent fluctuations in equation (3.14), the emitted radiative power is not affected and can be computed from the average field of temperature. 
Understanding and quantifying when turbulent fluctuations affect the mean absorbed radiative power is a much more complex task and is out of the scope of the present study. Nonetheless, for all cases studied here (A_R, B_R and C_R), no effects of temperature fluctuations in the mean radiative power have been observed, including on the cold side where radiative absorption phenomena are dominant in the boundary buffer layer (see figure $9(\mathrm{a}))$.

It is demonstrated in Appendix B that, in gaseous flows, the level of temperature fluctuations in turbulent boundary layers can be larger than the one observed in the studied cases for strongly heating or cooling systems but it does not exceed $30 \%$ and it decreases with Reynolds number. Moreover, coupling with radiation makes these temperature fluctuations smaller. Therefore, the absence of effects of temperature fluctuations on the mean radiative power is not necessarily limited to the present conditions and concerns a wide range of turbulent boundary layer conditions. In fact, much stronger interactions between turbulence and radiation are observed in combustion applications (Coelho 2012) where the heat release stemming from chemical reactions greatly enhances temperature fluctuations.

Although the mean radiative power is not influenced by temperature fluctuations in the present case, fluctuations of the radiative power field modify the balance in transport equations of enthalpy variance and turbulent heat flux, equations (3.4) and (3.8), and hence interact with the mean temperature field indirectly. In order to compute the relevant radiative power root-mean-square $P^{R}$ rms, it is necessary to subtract the standard error of the Monte-Carlo method from the total rms data. This specific treatment is only required for $P^{R}$ rms. Indeed, since the Monte-Carlo method is a stochastic approach, the computed time-averaged variance of radiative power is

$$
\overline{P^{R^{\prime 2}}}=\overline{P_{\mathrm{phys}}^{R^{\prime 2}}}+\overline{P_{\mathrm{err}}^{R^{2}}}+2 \overline{P_{\mathrm{phys}}^{R^{\prime}} P_{\mathrm{err}}^{R}},
$$

where the instantaneous radiative power $P^{R}=P_{\mathrm{phys}}^{R}+P_{\mathrm{err}}^{R}$ is composed of the real physical value of radiative power, $P_{\text {phys }}^{R}$, that is estimated by the Monte-Carlo method with a controlled error, and of a stochastic error $P_{\mathrm{err}}^{R}$. Assuming independency, between physical and stochastic fluctuations, the root-mean-square of radiative power is computed as

$$
\left(P_{\mathrm{rms}}^{R}\right)^{2}=\overline{P_{\mathrm{phys}}^{R^{\prime 2}}}=\overline{P^{R^{\prime 2}}}-\overline{P_{\mathrm{err}}^{R^{2}}}
$$

using the statistical estimation of $\overline{P_{\mathrm{err}}^{R^{2}}}$ provided by the Monte-Carlo approach. The profiles of radiative power root-mean-square $P^{R}{ }_{\text {rms }}$ and of the stochastic error $\left(\overline{P_{\mathrm{err}}^{R^{2}}}\right)^{1 / 2}$ are plotted in figure 9 (b). It has been verified by performing Monte-Carlo simulation on one snapshot with a higher accuracy and by averaging in homogeneous directions that profiles of averaged physical quantities are not modified. The chosen accuracy for coupled simulations is therefore good enough and a specific treatment is only necessary to extract the physical rms of radiative power. Contrary to the mean radiative power that abruptly vanishes away from the walls, fluctuations of radiative power do not disappear and their magnitude presents only slight variations for $y^{+}>20$.

As shown previously, the fluctuating radiative power introduces a new radiative dissipation term in equation (3.4) that is written as $\overline{\mathrm{h}^{\prime \prime} P^{R^{\prime}}}$. Since radiative power is the difference between absorbed and emitted powers, $P^{R}=P_{a}^{R}-P_{e}^{R}$, the radiative dissipation term is also composed of two terms:

$$
\overline{\mathrm{h}^{\prime \prime} P^{R^{\prime}}}=\overline{\mathrm{h}^{\prime \prime} P_{a}^{R^{\prime}}}-\overline{\mathrm{h}^{\prime \prime} P_{e}^{R^{\prime}}} .
$$

As explained by Ammouri et al. (1994), positive (resp. negative) fluctuations of enthalpy 
yields a larger (resp. smaller) emitted radiative power. Therefore, radiative emission always leads to a negative contribution to the total radiative dissipation term. On the other hand, the absorbed part is mainly positive. The radiative dissipation could then be positive in some conditions. However, the corresponding correlation coefficient between enthalpy and radiative power shown in figure 10 (a) is negative and indicates that fluctuations of radiative power are fairly anti-correlated with local fluctuations of enthalpy or temperature, especially close to the wall. The radiative dissipation is then here dominated by its emission part.

In the balance equation (3.8) of the turbulent heat flux, the term $\overline{v^{\prime \prime} P^{R^{\prime}}}$ related to the correlation coefficient shown in figure 10 (b) appears when radiation is taken into account. In the present case, this term is positive in the whole computational domain although there is no reason to generalize this result. It is worth noticing, by introducing the radiative energy flux, that this term can be split into two terms as done for the conductive heat flux in equation (3.8):

$$
\overline{v^{\prime \prime} P^{R^{\prime}}}=\overline{v^{\prime \prime} \frac{\partial q^{R^{\prime}}}{\partial x_{i}}}=-\frac{\partial}{\partial x_{i}}\left(\overline{q_{i}^{R^{\prime}} v^{\prime}}\right)+\overline{q_{i}^{R^{\prime}} \frac{\partial v^{\prime}}{\partial x_{i}}}
$$

It seems interesting to understand the sign of the term $\overline{v^{\prime \prime} P^{R^{\prime}}}$ in view of the fair correlation between enthalpy and radiative power fluctuations. Indeed, one could argue that the fluctuation of wall-normal velocity leads to a fluctuation of enthalpy due to convection which finally generates a variation in the radiative power. In order to verify this effect, a first attempt could consist in passing over the convected enthalpy fluctuations and directly approximate the variation of radiative power as a result from convection and the shape of the mean profile $\overline{P^{R}}$. Turbulent diffusion of the mean radiative power with a gradient assumption would then lead to

$$
\overline{v^{\prime \prime} P^{R^{\prime}}} \propto-\frac{\partial \overline{P^{R}}}{\partial x_{i}}
$$

Although this approximation gives the right sign for the term $\overline{v^{\prime \prime} P^{R^{\prime}}}$ in the very near wall region on both sides, it becomes erroneous for $y^{+}>25$ where the gradient of the mean radiative power profile changes sign. Skipping the enthalpy fluctuations to understand the correlation between wall-normal velocity and the radiative power is therefore not appropriate.

Another approach is proposed by momentarily considering the fourth-order crossmoment $\overline{v^{\prime \prime} \mathrm{h}^{\prime \prime} \mathrm{h}^{\prime \prime} P^{R^{\prime}}}$ which introduces enthalpy fluctuations as the vehicle that could explain the correlation mechanism between velocity and radiative power variations. Two high-order correlation coefficients $C_{1}$ and $C_{2}$ are then defined as

$$
\begin{aligned}
& \overline{v^{\prime \prime} \mathrm{h}^{\prime \prime} \mathrm{h}^{\prime \prime} P^{R^{\prime}}}=C_{1} \widetilde{v^{\prime \prime} \mathrm{h}^{\prime \prime}} \overline{h^{\prime \prime} P^{R^{\prime}}} \\
& \overline{v^{\prime \prime} \mathrm{h}^{\prime \prime} \mathrm{h}^{\prime \prime} P^{R^{\prime}}}=C_{2} \widetilde{\mathrm{h}^{\prime \prime} \mathrm{h}^{\prime \prime}} \overline{v^{\prime \prime} P^{R^{\prime}}}
\end{aligned}
$$

Finally, the term $\overline{v^{\prime \prime} P^{R^{\prime}}}$ can be indirectly related to the high-order statistical moment by writing

$$
\overline{v^{\prime \prime} P^{R^{\prime}}}=C \frac{\widetilde{v^{\prime \prime} \mathrm{h}^{\prime \prime}} \overline{\mathrm{h}^{\prime \prime} P^{R^{\prime}}}}{\widetilde{\mathrm{h}^{\prime \prime} \mathrm{h}^{\prime \prime}}},
$$

where $C=C_{1} / C_{2}$ is computed from equation (3.22) and plotted in figure 11. The profile of the coefficient $C$ is regular, positive and varies from approximatively unity close to the walls to 2.7 in the core of the channel. Neglecting differences between Favre and Reynolds 


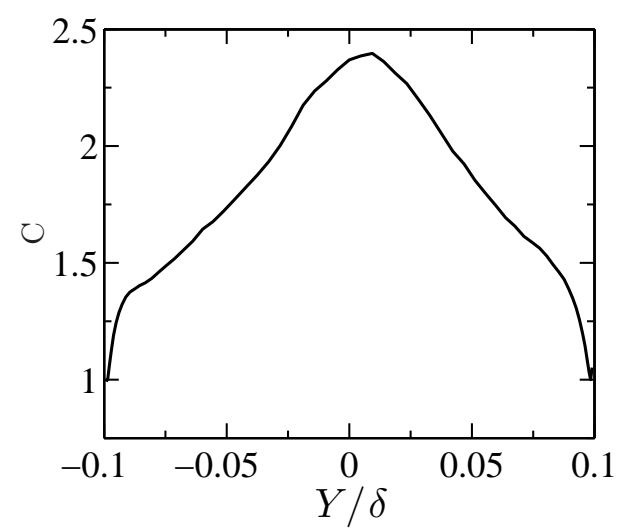

Figure 11. Correlation coefficient defined in equation (3.22) in case A_R.

fluctuations and inserting truncated Taylor series of $v^{\prime}=a y^{2}, \mathrm{~h}^{\prime}=b y$ and $P^{R^{\prime}}=c$ in the definition of $C$ gives

$$
C=\frac{\overline{b^{2}} \overline{a c}}{\overline{a b} \overline{b c}}
$$

The fact that $C=1$ at walls indicates that $c$ is proportional to $b$ : Fluctuations of radiative power very close to the wall are mainly determined by local fluctuations of temperature.

Since the radiative dissipation term $\overline{\mathrm{h}^{\prime \prime} P^{R^{\prime}}}$ is negative, it can then be postulated from equation (3.22) that the term $\overline{v^{\prime \prime} P^{R^{\prime}}}$ appearing in equation (3.8) always has the opposite sign of the turbulent flux $\bar{\rho} v^{\prime \prime} \mathrm{h}^{\prime \prime}$. In the present configuration, this explains the positive sign of the correlation between wall-normal velocity and radiative power. As the term $\overline{v^{\prime \prime} P^{R^{\prime}}}$ presents a sign opposite to the one of the turbulent heat flux, it therefore appears as a loss term in equation (3.8). This loss mechanism described by equation (3.22) can be summarized as follows: A positive or negative variation of wall-normal velocity generates a fluctuation of enthalpy whose sign is determined by the direction of the turbulent heat flux; And this enthalpy fluctuation then induces a variation of opposite sign in radiative power.

\subsubsection{Summary of radiation effects in turbulent channel flow}

The coupling between the temperature and radiative fields has been carefully detailed through the analysis of DNS/Monte-Carlo results obtained in cases A and A_R. Figure 12 sums up the different interactions between Favre or Reynolds mean fields and fluctuating fields of velocity, temperature and radiation observed in the present channel flow simulations.

The feedback of mean temperature on velocity is done through the variation of temperaturedependent properties such as mean density and dynamic viscosity. In cases A and A_R characterized by a relative small difference of wall temperatures, this effect is negligible although it is effective in cases with larger temperature difference (Zhang et al. 2013a). On the other hand, impact of temperature fluctuations on the velocity field mainly through density variations is negligible in all investigated cases, making Reynolds and Favre averaging operations similar. In the specific configuration of a channel flow, convection by the mean velocity field does not influence the temperature field directly. It has obviously a major role in any general flow configuration. Besides, the fluctuations of velocity play a critical role in the obtained profiles of mean temperature and its root-mean-square through the turbulent heat flux and the production of enthalpy variance. 


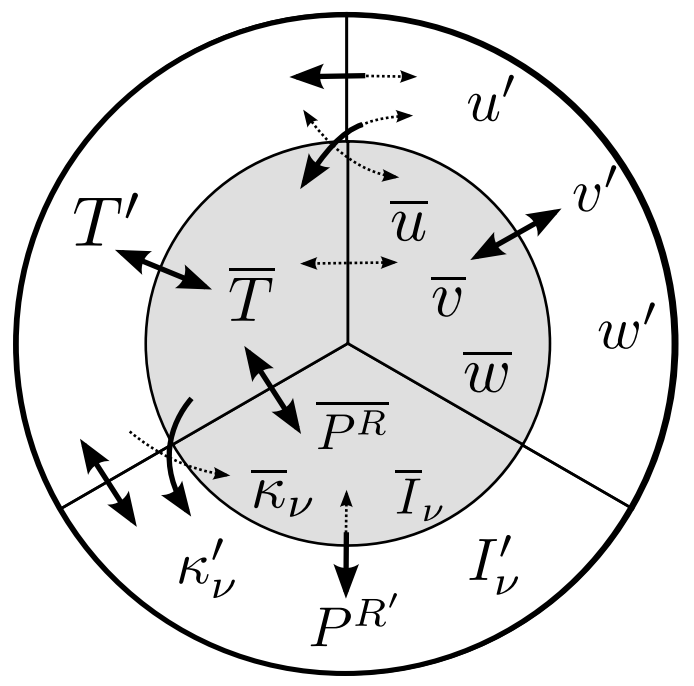

Figure 12. Scheme of coupling effects in turbulent channel flow with radiation. The fields of velocity $(u, v, w)$, temperature $(T)$ and radiative power $\left(P^{R}\right)$ are split into mean and fluctuating components. Other quantities are considered for the radiation field: The spectral intensity $\left(I_{\nu}\right)$ and absorption coefficient $\left(\kappa_{\nu}\right)$. Main effects are represented by thick plain arrows while thin dotted arrows indicate negligible or null effects.

As outlined in Fig 12, the mean temperature and its fluctuations are tightly connected through the turbulent heat flux and the production term in the enthalpy variance balance equation. Similarly, the mean temperature field and the mean radiative power strongly depend on each other. The aforementioned modification of production in equation (3.4) is an indirect effect of radiation involving the two-way interaction between $\overline{P^{R}}, \bar{T}$ and $T^{\prime}$.

When radiation is accounted for, additional loss terms appear in the balance equations of enthalpy variance and turbulent heat flux, which make turbulent transport of the mean temperature field less efficient. The level of fluctuations of radiative fields is also determined from the magnitude of mean temperature and mean radiative fields.

Finally, it has been observed that the influence of fluctuating temperature and radiative fields is not noticeable on the mean radiative field. Such a property has been assumed in the development of a wall model for large-eddy simulations by Zhang et al. (2013b) to account for radiation effects within the inner structure of turbulent boundary layers.

\subsection{Effects of wall temperature difference and Reynolds number}

In this section, cases A, B and C are compared with and without accounting for radiation to enhance the effects of wall temperature difference and Reynolds number. Analysis of the results focuses on the turbulent heat flux, the enthalpy root-mean-square and the main contributing terms in their balance equations. Radiation effects prevent collapsing of scaled profiles when standard wall-scaling is used. A new scaling is proposed to improve collapsing of several profiles.

\subsubsection{Effect of wall temperature difference}

In comparison to the cases A and A_R, cases B and B_R are characterized by a large wall temperature difference (see table 1). Mean temperature profiles of these four cases are shown in figure 13. Small differences between cases A and B without radiation are due to noticeable effects of mean density variations. When accounting for radiation, the 




(a)

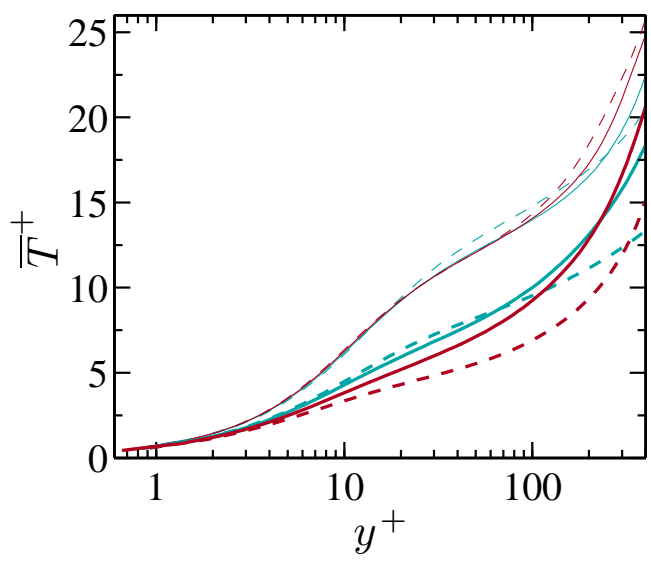

(b)

Figure 13. Profiles of mean temperature scaled by wall temperatures (a) and in wall units (b) on the cold side (blue color) and hot side (red color) in cases A (thin plain line), A_R (thick plain line), B (thin dashed line) and B_R (thick dashed line).



(a)



(b)

Figure 14. Profiles of turbulent kinetic energy on the cold side (blue color) and hot side (red color) in cases A (thin plain line), A_R (thick plain line), B (thin dashed line) and B_R (thick dashed line): (a) $k^{+}$, scaled with standard wall-scaling. (b) $k^{*}$, scaled with semi-local scaling.

modification of the mean temperature in both $\bar{T}$ and $\bar{T}^{+}$profiles is stronger between case B and B_R because of the amplification of radiative effects at high temperature.

Profiles of mean velocity reported by Zhang et al. (2013a) exhibit a more significant impact of the mean density in cases B and B_R. The turbulent kinetic energy in wall units $k^{+}=\widetilde{u_{i}^{\prime \prime} u_{i}^{\prime \prime}} /\left(2 u_{\tau}^{2}\right)$ is plotted in figure 14 (a). The variations of mean density prevent collapsing of the profiles, especially when comparing cases with small and large wall temperature difference. The effect of radiation on $k^{+}$, associated with the changes in mean temperature profile and hence mean density, is small.

In order to improve the agreement between profiles corresponding to different cases, a semi-local scaling is applied: Non-uniformity of gas properties is considered by using local values of density, viscosity and thermal capacity (Huang et al. 1995; Coleman et al. 1995; Dailey et al. 2003). The corresponding friction velocity $u_{\tau}^{*}$ and friction temperature 


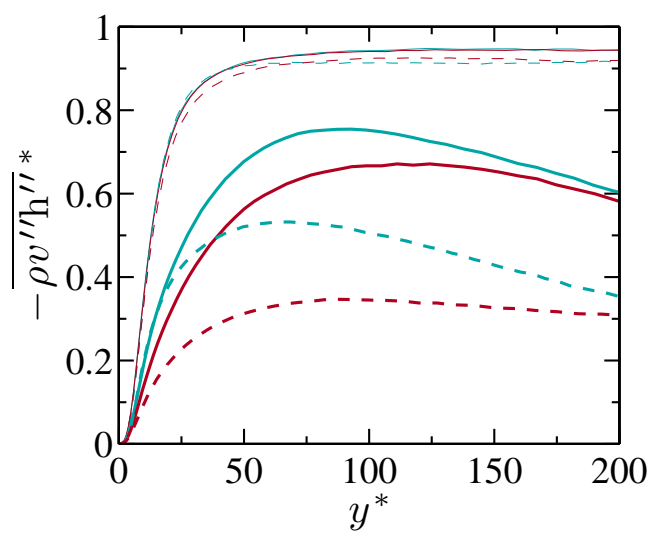

(a)



(c)

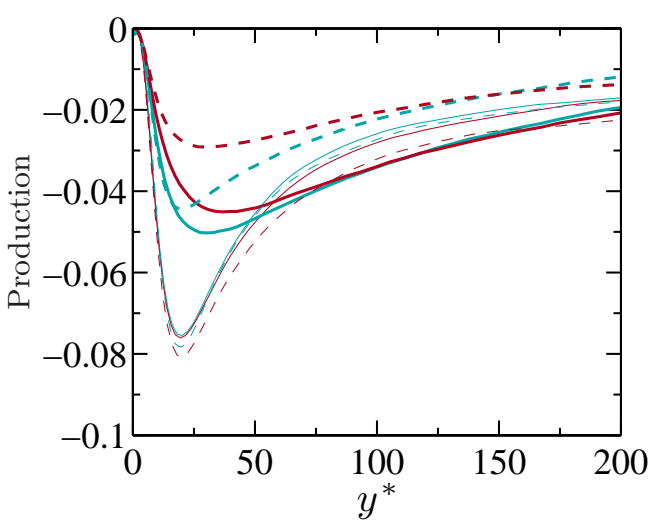

(b)



(d)

FIGURE 15. Profiles of turbulent heat flux (a) and its production term (b), EPG term (c) and correlation between velocity and radiative power (d) in semi-local scaled units on the cold side (blue color) and hot side (red color) in cases A (thin plain line), A_R (thick plain line), B (thin dashed line) and B_R (thick dashed line).

$T_{\tau}^{*}$ are then defined as:

$$
u_{\tau}^{*}=\left(\frac{\overline{\tau_{w}}}{\bar{\rho}}\right)^{1 / 2}, \quad T_{\tau}^{*}=\frac{\left|\overline{q_{w}^{c d}}\right|}{\bar{\rho} \overline{c_{p}} u_{\tau}^{*}},
$$

and the non-dimensional wall distance $y^{*}$ is given by

$$
y^{*}=\frac{\bar{\rho} y u_{\tau}^{*}}{\bar{\mu}}
$$

The turbulent kinetic energy in semi-local coordinates $k^{*}=k / u_{\tau}^{* 2}$ is shown in figure 14 (b). Collapsing of $k^{*}$ profiles is significantly improved, even for cases with radiation. Scaling of variables enables some cases variability to be filtered out, and the collapsing of their profiles outlines that the same underlying physical phenomenon is responsible for the shape of these profiles. Here, collapsing of $k^{*}$ curves confirms that radiation affects turbulent kinetic energy indirectly through the change of temperature-dependent gas properties. Henceforward, semi-local scaling is used to normalize turbulent quantities. 
The scaled turbulent heat flux, issued from the semi-local scaling,

$$
{\overline{\rho v^{\prime \prime} \mathrm{h}^{\prime \prime}}}^{*}=\frac{\widetilde{\rho} v^{\prime \prime} \mathrm{h}^{\prime \prime}}{\bar{\rho} u_{\tau}^{*} \overline{c_{p}} T_{\tau}^{*}}=\frac{\widetilde{\rho v^{\prime \prime} \mathrm{h}^{\prime \prime}}}{\left|\overline{q_{w}^{c d}}\right|}
$$

does not differ from the one obtained with standard wall-scaling. Profiles of $\overline{\rho v^{\prime \prime} \mathrm{h}^{\prime \prime}}$ * are compared in figure 15 (a). Good agreement between results of $\mathrm{A}$ and $\mathrm{B}$ is obtained. However, cases with radiation A_R and B_R are significantly different. Radiation effects are strongly dependent on temperature and non-linear, which explains why the deviation from cases without radiation is larger in case B_R because of higher temperatures.

The three dominant terms in the balance equation (3.8) of the turbulent heat flux, i.e. production, enthalpy-pressure-gradient correlation term (EPG) and radiation term, are also reported in figure 15. Following the semi-local scaling approach, these quantities are scaled by $\left|\overline{q_{w}^{c d}}\right| \overline{\tau_{w}} / \mu$. The collapsing of production and EPG term profiles corresponding to cases A and B is good except for the hot side in case B which presents a small difference that could not be corrected entirely by semi-local scaling.

In cases with radiation, the scaled production that is related to $-\widetilde{v^{\prime \prime} v^{\prime \prime}} * \frac{d \tilde{h}^{*}}{d y^{*}}$ is smaller in magnitude. Since, as shown for the turbulent kinetic energy $k$, the semi-local scaled root-mean-square of wall-normal velocity $\widetilde{v^{\prime \prime} v^{\prime \prime}} *$ is barely affected by radiation through the mean density variation, the modification of the production magnitude is due to the change of scaled mean enthalpy gradient $\frac{d \tilde{\mathrm{h}}^{*}}{d y^{*}}$ that is tightly related to $\frac{d \bar{T}^{+}}{d y^{+}}$. Indeed, for $y^{+}$between 10 and 30 where the production peaks, the variations between cases in the gradient of the scaled mean temperature (related to the slope of $\bar{T}^{+}\left(y^{+}\right)$plotted in figure 13 (b)) agree with the differences observed for the peak value of the scaled production term. The differences of the wall-scaled enthalpy gradient are therefore responsible for the variations in the production term shown in figure 15 (b).

The enthalpy-pressure-gradient correlation term appearing in the transport equation of the turbulent heat flux is shown in figure 15 (c). It mostly compensates production for $y^{*}<100$, and therefore the same variations between cases is retrieved. The last of the three dominant terms for cases with radiation in equation (3.8) is the scaled correlation between wall-normal velocity and radiative power $\overline{v^{\prime \prime} \mathrm{P}^{R^{\prime}}}$. It is shown in figure 15 (d). The fact that $\overline{v^{\prime \prime} \mathrm{P}^{R^{\prime}}}$ is larger in case A_R is not obvious as the magnitude of this radiative loss term is determined by multiple phenomena as described in section 3.1.4.

\subsubsection{Radiation-based scaling}

It has been shown that semi-local scaling works satisfactorily for cases without radiation by accounting for the effects of variable fluid properties. However, it cannot account for the specific role of radiation. Each case appears specific, although the same physical interpretation can explain the shapes and magnitudes of the observed profiles, as seen for the production term in equation (3.8). A new scaling is therefore derived to account for the variability of radiation effects.

As the mean energy balance equation without radiation is

$$
\overline{q_{y}^{c d}}(Y)+\widetilde{\rho} \widetilde{v^{\prime \prime} \mathrm{h}^{\prime \prime}}(Y)=\overline{q_{w}^{c d}} .
$$

the turbulent heat flux is equal to the wall conductive flux $\overline{q_{w}^{c d}}$ outside of the viscous sublayer, where the conductive heat flux is negligible. The wall conductive flux $\overline{q_{w}^{c d}}$ is then the right quantity for scaling of the turbulent heat flux and to define the friction temperature. But, when radiation is taken into account, the mean energy balance equation 


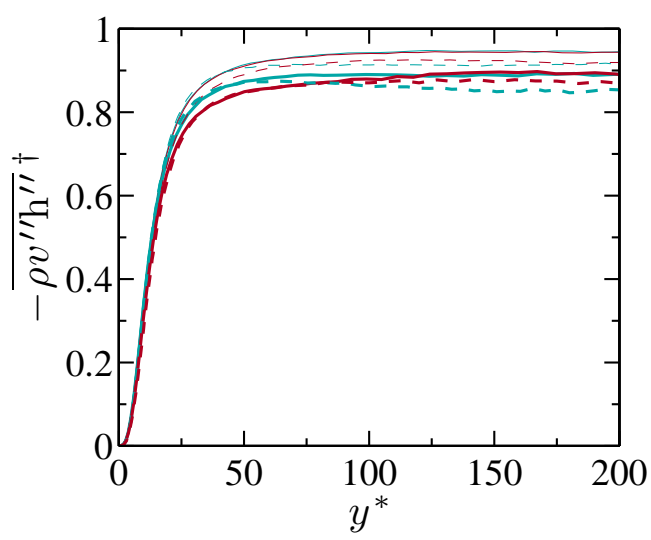

(a)

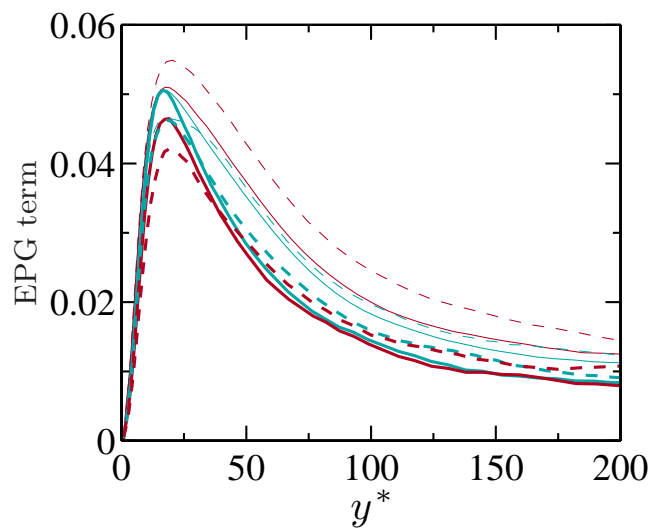

(c)



(b)



(d)

FIGURE 16. Profiles of turbulent heat flux (a) and its production term (b), EPG term (c) and correlation between velocity and radiative power (d) using radiation-based scaling on the cold side (blue color) and hot side (red color) in cases A (thin plain line), A_R (thick plain line), B (thin dashed line) and B_R (thick dashed line).

(3.7) becomes

$$
\overline{q_{y}^{c d}}(Y)+\widetilde{\rho} \widetilde{v^{\prime \prime} \mathrm{h}^{\prime \prime}}(Y)=\overline{q_{w}^{c d}}-\Delta \overline{q^{R}}(Y) \equiv \overline{q^{\dagger}}(Y),
$$

where $\Delta \overline{q^{R}}(Y)$ is the difference between the local radiative flux and the wall radiative flux,

$$
\Delta \overline{q^{R}}(Y)=\overline{q^{R}}(Y)-\overline{q_{w}^{R}}
$$

The radiative fluxes $\overline{q^{R}}(Y)$ and $\overline{q_{w}^{R}}$ both contain the wall-wall radiative flux $\overline{q_{w w}^{R}}$ exchanged between the walls through the whole gaseous medium, that does not participate to the fluid energy balance and is zero if the walls are at the same temperature. This contribution is then suppressed in the definition of $\Delta \overline{q^{R}}(Y)$.

Similarly to the wall conductive flux $\overline{q_{w}^{c d}}$ in equation $(3.27),\left|\overline{q^{\dagger}}(Y)\right|$ is the right quantity for scaling of the turbulent heat flux for cases involving radiation. The sum of conductive and turbulent heat flux, equal to $\overline{q^{\dagger}}(Y)$, is a function of wall distance whose shape is determined by the intensity and nature of radiative energy transfer. For cases without radiation, $\overline{q^{\dagger}}(Y)$ turns back to the wall conductive flux.

The turbulent heat flux, normalized by $\left|\overline{q^{\dagger}}(Y)\right|,{\overline{\rho v^{\prime \prime} \mathrm{h}^{\prime \prime}}}^{\dagger}$ is plotted in figure 16 (a). In 
comparison with figure 15 (a), the agreement between the different cases is significantly improved. Thanks to the radiation-based scaling, the scaled turbulent heat flux represents the relative strength of turbulent transport compared to conduction for all cases. For this reason, all profiles now present the same shape and reach a plateau. The remaining discrepancies concern the level of this plateau that is related to the remaining contribution of the conductive heat flux which, in the studied configurations with hot and cold walls, does not tend to zero in the core of the channel. The level of the plateau is then determined by the mean temperature gradient at the center of the channel and is different for each case. It is expected that, for symmetrical channel flows with the same wall temperatures or for external boundary layers, this effect is not present, making the proposed scaling even more efficient.

The three main terms in the balance equation of turbulent heat flux are also shown in figure 16, where the production, EPG contribution and radiative term are now scaled by $\left|\overline{q^{\dagger}}(Y)\right| \overline{\tau_{w}} / \mu$. The agreement between profiles is also much better when the radiationbased scaling is considered. In order to understand the effect of the scaling, the scaled production term is written as

$$
\frac{-\overline{\rho v^{\prime \prime} v^{\prime \prime}} \frac{\mathrm{d} \widetilde{\mathrm{h}}}{\mathrm{d} y}}{\overline{\mid q^{\dagger}}(Y) \mid \overline{\tau_{w}} / \mu} \approx-\overline{\rho v^{\prime \prime} v^{\prime \prime}} * \operatorname{Pr}\left(1-\overline{\rho v^{\prime \prime} \mathrm{h}^{\prime \prime}}{ }^{\dagger}\right)
$$

where $\frac{\mathrm{d} \widetilde{\mathrm{h}}}{\mathrm{d} y} \approx \overline{c_{p}} \frac{\mathrm{d} \widetilde{T}}{\mathrm{~d} y}$ is expressed from the mean energy balance equation. The improved agreement between scaled profiles of production is then issued from the obtained agreement between profiles of the turbulent heat flux. Discrepancies are nonetheless more noticeable in figure 16 (b) because the term $\left(1-\overline{\rho v^{\prime \prime} \mathrm{h}^{\prime \prime}}{ }^{\dagger}\right)$ outlines the aforementioned plateau mismatch. As the radiation-based scaling improves the collapsing of production profiles, its main counter-balancing terms, that are the contribution and radiation term, benefit from the same effect although the improved collapsing of profiles is not striking for the latter.

The enthalpy root-mean-square and the main terms in the enthalpy variance balance equation (3.4), production, molecular and radiative dissipations, are scaled using semilocal scaling and shown in figure 17. The transport equation terms are therefore scaled by ${\overline{q_{w}^{c d}}}^{2} / \bar{\mu}$. Once again, the good collapsing of profiles corresponding to cases without radiation demonstrate the efficiency of semi-local scaling to account for variable flow properties effects. Nonetheless, when radiation is taken into account, the rms of enthalpy, its production and molecular dissipation are totally different. As shown previously, then deviation from cases without radiation is stronger for higher temperatures.

The same results using radiation-based scaling are presented in figure 18. Defining a radiation-based friction temperature as

$$
T_{\tau}^{\dagger}=\frac{\left|\overline{q^{\dagger}}(Y)\right|}{\bar{\rho} \overline{c_{p}} u_{\tau}}
$$

the normalized enthalpy $\mathrm{h}_{\mathrm{rms}}^{\dagger}$ is scaled by $\overline{c_{p}} T_{\tau}^{\dagger}$ while the balance equation terms are scaled by the quantity $\overline{q^{\dagger}}(Y)^{2} / \bar{\mu}$. The radiation-based scaling allows similar shapes of $\mathrm{h}_{\mathrm{rms}}^{\dagger}$ to be retrieved for all cases. Corresponding cases with and without radiation seem to collapse, although case B_R deviates from case A_R for $y^{*}>75$. As the proposed radiation-based scaling accounts for variations of the turbulent heat flux profiles, the fact that the enthalpy rms does not directly depend on the turbulent heat flux can 


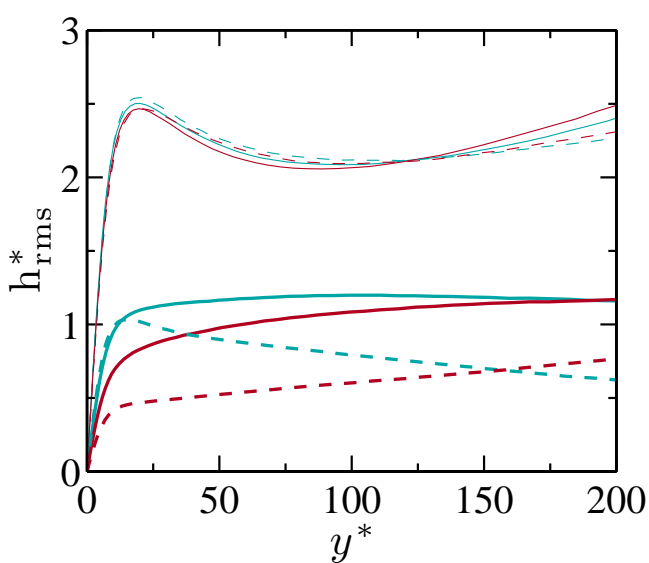

(a)



(c)

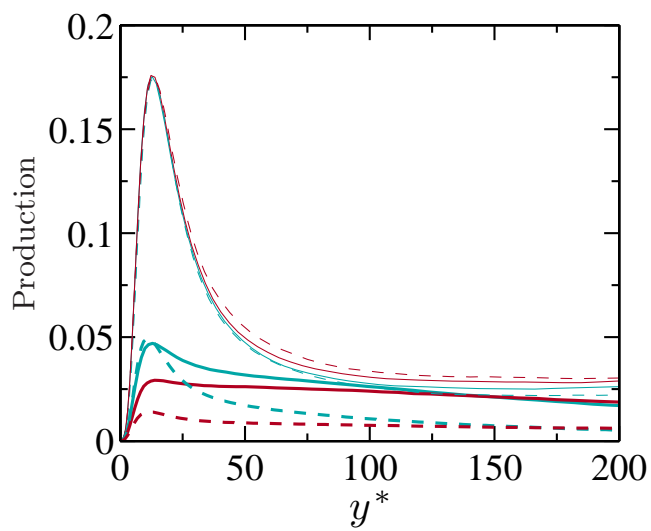

(b)



(d)

Figure 17. Profiles of enthalpy rms (a) and its production term (b), molecular dissipation (c) and radiative dissipation (d) using semi-local scaling on the cold side (blue color) and hot side (red color) in cases A (thin plain line), A_R (thick plain line), B (thin dashed line) and B_R (thick dashed line).

explain these discrepancies. On the other hand, the scaled production that is written as

$$
\frac{-\bar{\rho} \widetilde{v^{\prime \prime} \mathrm{h}^{\prime \prime}} \frac{\mathrm{d} \widetilde{\mathrm{h}}}{\mathrm{d} y}}{\overline{q^{\dagger}}(Y)^{2} / \mu} \approx-\overline{\rho v^{\prime \prime} \mathrm{h}^{\prime \prime}} \dagger \operatorname{Pr}\left(1-\overline{\rho v^{\prime \prime} \mathrm{h}^{\prime \prime}}{ }^{\dagger}\right),
$$

simply depends on the turbulent heat flux and the obtained agreement between production profiles in figure 18 (b) is better. Production of enthalpy variance is mainly compensated by molecular and radiative dissipations which then individually show a fair agreement of their profiles using the new scaling. As radiation introduces an additional and significant loss term in the balance equation of enthalpy variance, the balance between production and molecular dissipation is strictly different between cases with and without radiation. That is why the agreement between profiles of molecular dissipation in figure 18 (c) is here correct only between cases with or without radiation, separately. However, switching progressively from a case without radiation to cases with more and more significant radiative effects, a continuous transition is expected. This trend is more visible in other studied cases and is detailed in the next section that deals with Reynolds number effects. 


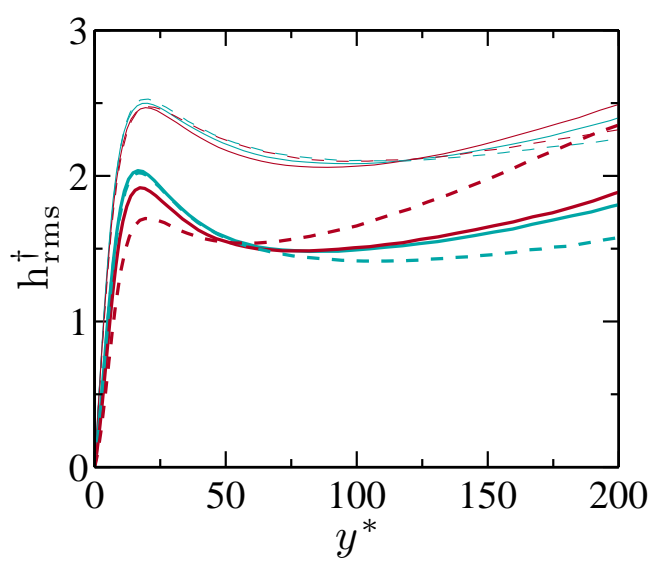

(a)

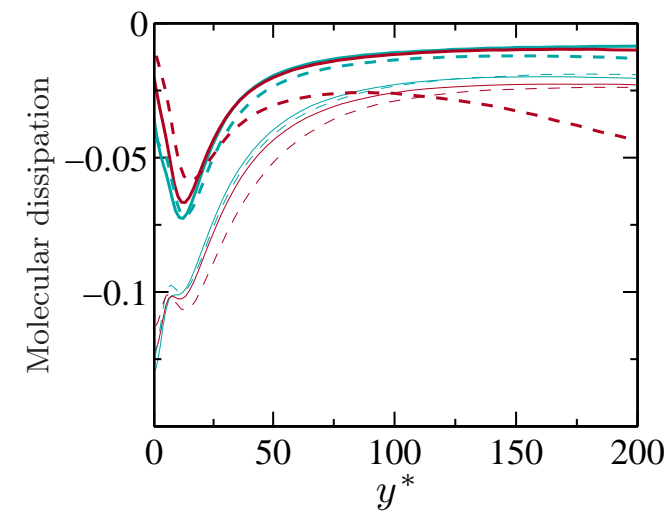

(c)



(b)

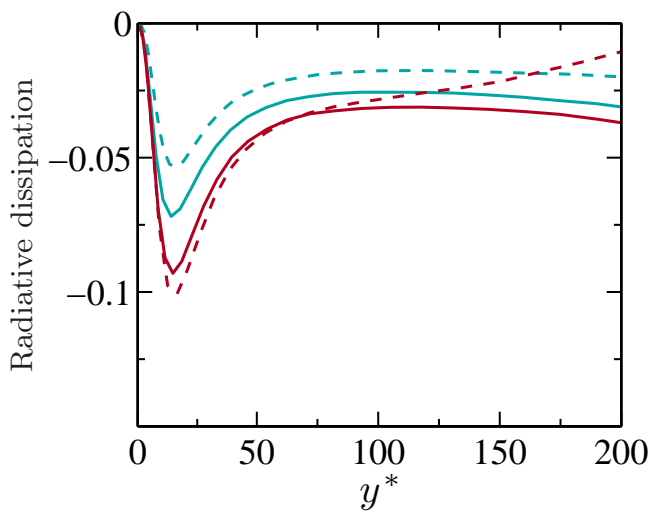

(d)

Figure 18. Profiles of enthalpy rms (a) and its production term (b), molecular dissipation (c) and radiative dissipation (d) using radiation-based scaling on the cold side (blue color) and hot side (red color) in cases A (thin plain line), A_R (thick plain line), B (thin dashed line) and B_R (thick dashed line).

Finally, it is worth noticing that the use of the radiation-based friction temperature $T_{\tau}^{\dagger}$ is not sufficient to improve the agreement between mean temperature profiles (see figure 19). Introducing the model of the turbulent diffusivity, the turbulent heat flux is related to the gradient of mean temperature. The mean energy balance equation in equation (3.28) also further constrain the relation between $\overline{\rho v^{\prime \prime} \mathrm{h}^{\prime \prime}}$ and the conductive flux, linked to the gradient of mean temperature. Therefore, the radiation-based friction temperature $T_{\tau}^{\dagger}$ can in fact properly scale the gradient of mean temperature for the different cases as it did for the turbulent heat flux. However, the radiation-based friction temperature $T_{\tau}^{\dagger}$ depends on the wall distance and this prevents it from appearing straightforwardly in the expression of the mean temperature after integration of the gradient $\mathrm{d} \bar{T} / \mathrm{d} y$.

\subsubsection{Effect of Reynolds number}

Figure 20 compares cases A and A_R to cases C and C_R, characterized by a larger Reynolds number, in terms of mean temperature profiles. On the one hand, radiation effects are reduced by the increased influence of turbulent transport, which makes the temperature profile in case C_R closer to those of cases without radiation. On the other 


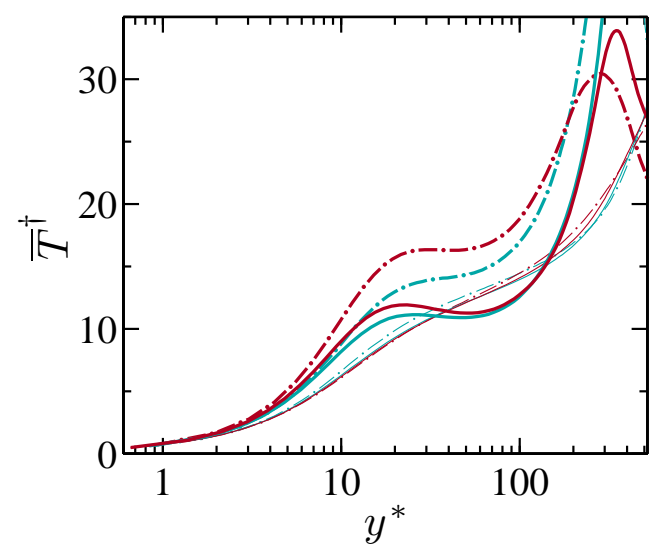

FiguRE 19. Profiles of mean temperature scaled by the radiation-based friction temperature $T_{\tau}^{\dagger}$ on the cold side blue color) and hot side (red color) in cases A (thin plain line), A_R (thick plain line), B (thin dashed line) and B_R (thick dashed line).



(a)



(b)

FiguRE 20. Profiles of mean temperature scaled by wall temperatures (a) and in wall units (b) on the cold side (blue color) and hot side (red color) in cases A (thin plain line), A_R (thick plain line), C (thin dashed-dotted line) and C_R (thick dashed-dotted line).

hand, a modification of the nature of radiative energy transfer has also been observed (Zhang et al. 2013a).

For cases $\mathrm{A}$ and $\mathrm{C}$ without radiation, a good agreement between profiles of mean turbulent heat flux is obtained by using semi-local scaling, as shown in figure 21. The same agreement is obtained for the main terms (production and EPG terms) in the balance equation of the turbulent heat flux. When radiation is accounted for in cases A_R and C_R, the profiles do not collapse on the ones of cases A and C. Nonetheless, results of case $C_{-} R$ sit between profiles of cases without radiation and the ones of case A_R, which is due to the weakened radiative effects at higher Reynolds number.

The same profiles are reported in figure 22 using the radiation-based scaling. As in section 3.2.2, agreement between profiles is significantly improved, except for the radiative term. It can be also noticed that results for case C_R remain between case A_R and case $\mathrm{A} / \mathrm{C}$.

Similar observations are obtained for the enthalpy root-mean-square and for its pro- 


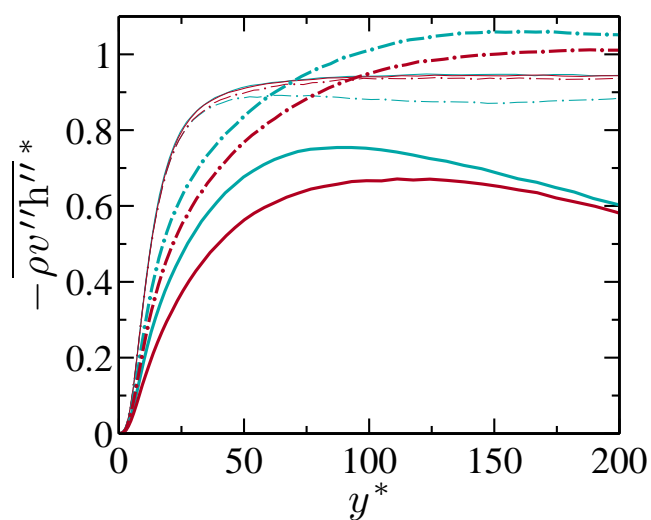

(a)

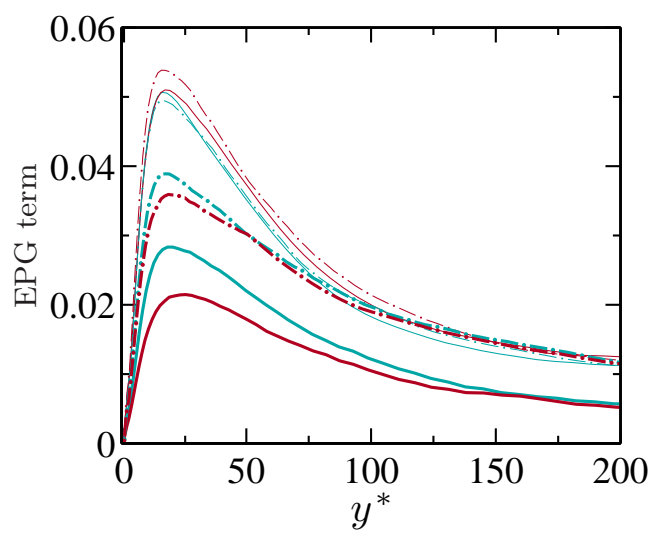

(c)

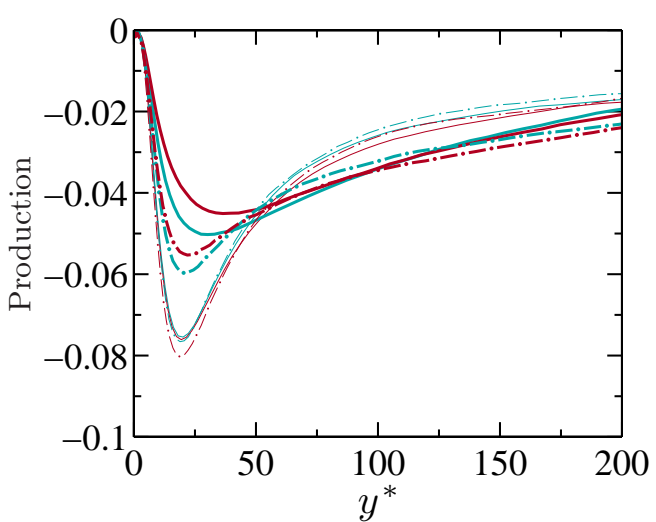

(b)

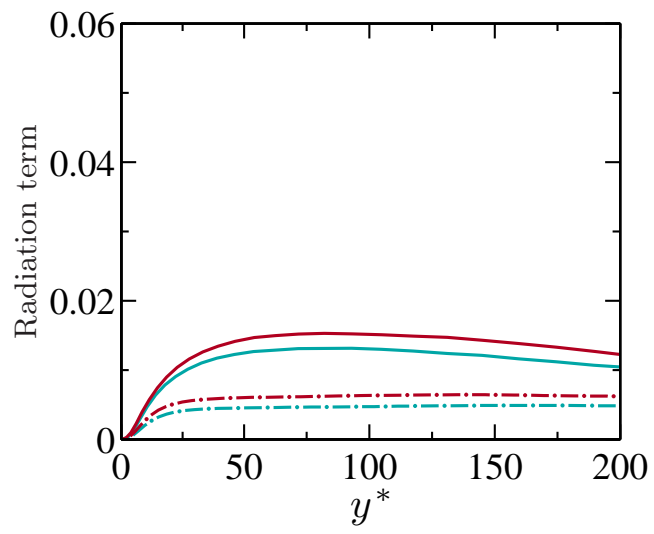

(d)

Figure 21. Profiles of turbulent heat flux (a) and its production term (b), EPG term (c) and correlation between velocity and radiative power (d) in semi-local scaled units on the cold side (blue color) and hot side (red color) in cases A (thin plain line), A_R (thick plain line), C (thin dashed-dotted line) and C_R (thick dashed-dotted line).

duction and dissipation terms shown in figure 23 and 24 using semi-local scaling and radiation-based scaling, respectively. Contrary to the comparison between cases A_R and B_R, it clearly appears in figure 24 (d) that the scaled molecular dissipation profiles exhibit a transition between cases without radiation and cases with stronger and stronger radiative energy transfer. Consequently, although profiles of production correctly collapse with the proposed radiation-based scaling, its necessary splitting into two dissipative terms prevents collapsing for molecular and radiative dissipation profiles separately. The balance between molecular and radiative dissipations is controlled by the importance of radiation so that a smooth transition between profiles of different cases can be expected when radiative energy transfer increases from being negligible to strongly dominant.

With the same arguments, the profiles of EPG and radiation terms in the balance equation of the turbulent heat flux cannot entirely collapse with the proposed radiationbased scaling. The balance between the two terms is determined by the weight of radiation effects.

For the sake of clarity and brevity, only high-pressure cases have been studied. In atmospheric conditions, similarly to the studied case $\mathrm{C}$ with a higher Reynolds number, the relative magnitude of radiative energy transfer compared to turbulent transport 


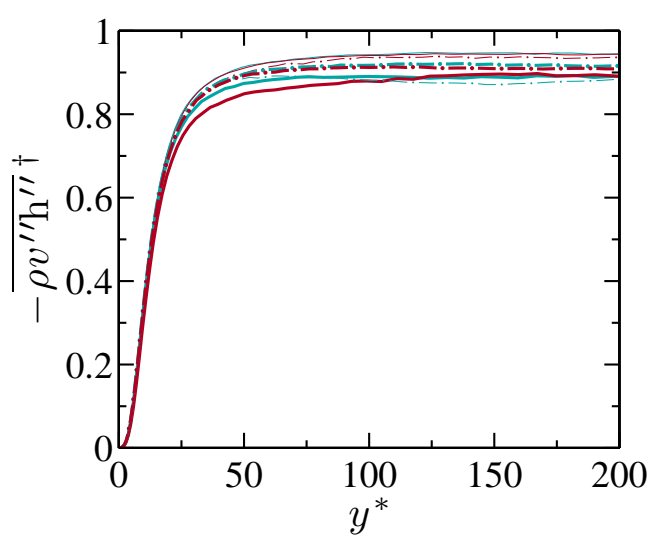

(a)

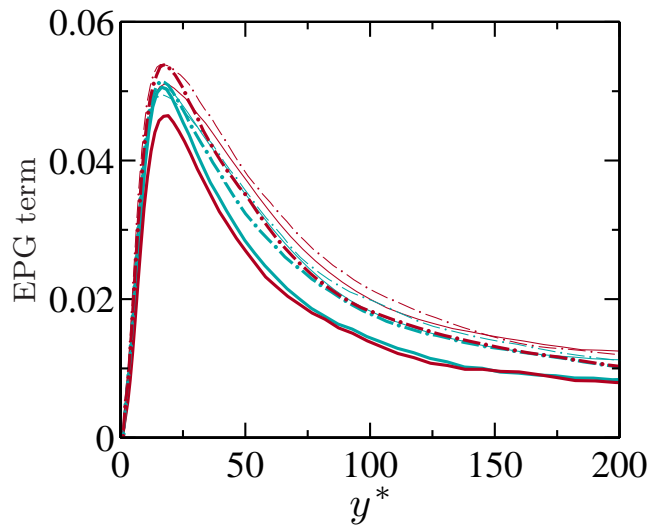

(c)

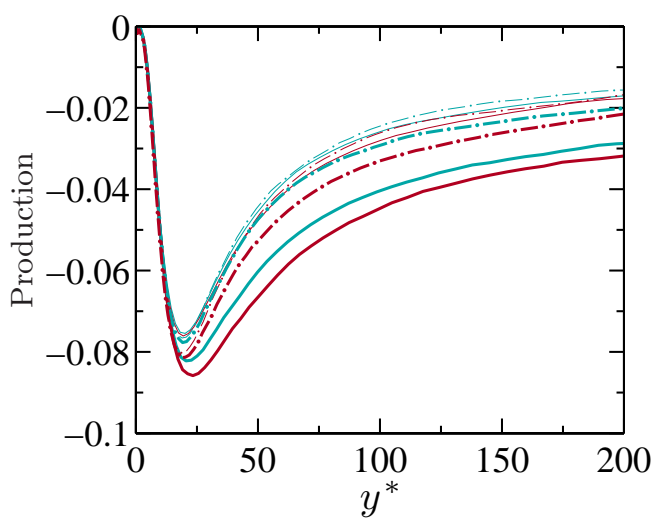

(b)

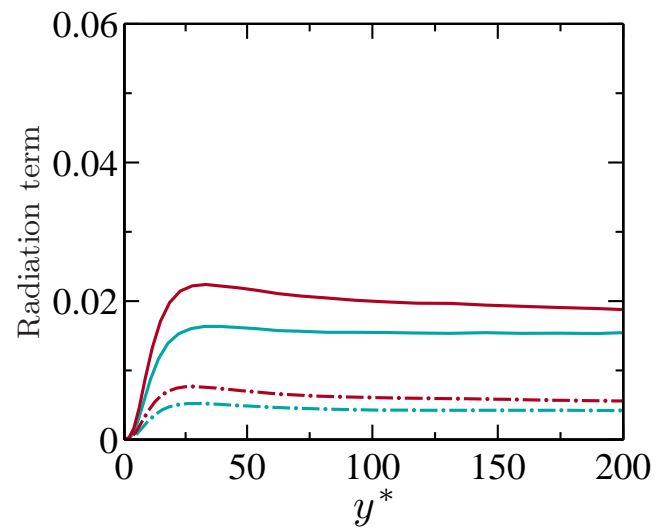

(d)

FIGURE 22. Profiles of turbulent heat flux (a) and its production term (b), EPG term (c) and correlation between velocity and radiative power (d) using radiation-based scaling on the cold side (blue color) and hot side (red color) in cases A (thin plain line), A_R (thick plain line), C (thin dashed-dotted line) and C_R (thick dashed-dotted line).

decreases. Compared to high pressure cases, profiles at atmospheric pressure are then closer to corresponding cases without radiation. The various effects of wall temperatures, wall emissivities, pressure and Reynolds number have been extensively studied on the mean temperature profile by Zhang et al. (2013a). As seen in the present study, no universal profile appears: Each case is different from the other. Additional effects that have not been investigated such as different reflection laws, possibly including spectral effects, would also yield to different profiles. Nonetheless, for all these cases that have not been studied here, the different additional terms due to radiation in the balance equations of enthalpy root-mean-square and turbulent heat flux remain and the associated physical interpretation derived here is expected to remain valid. This is especially true for the radiation-based scaling designed in equation (3.28) to properly scale the turbulent heat flux in any radiative conditions by accounting for the balance between the conductive heat flux and the turbulent heat flux only.

\subsection{Effects on the turbulent Prandtl number}

Previously shown results have outlined the effect of radiation on the turbulent heat flux. A simple model of turbulent heat transfer is based on the turbulent Prandtl number $\operatorname{Pr}_{t}$ 


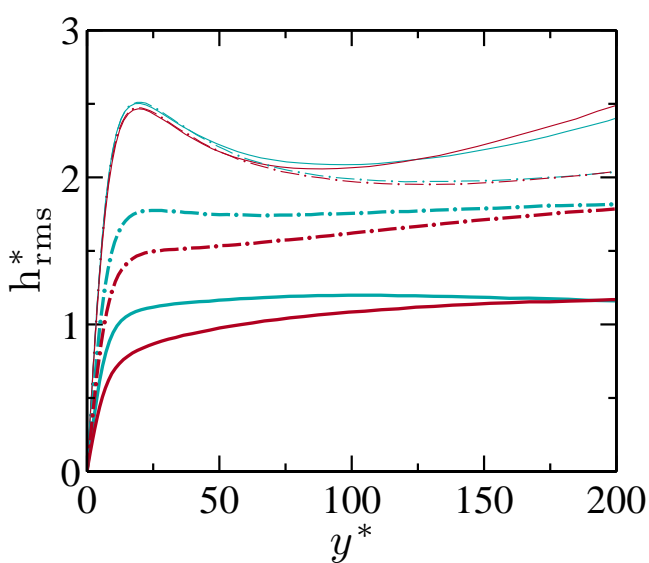

(a)



(c)

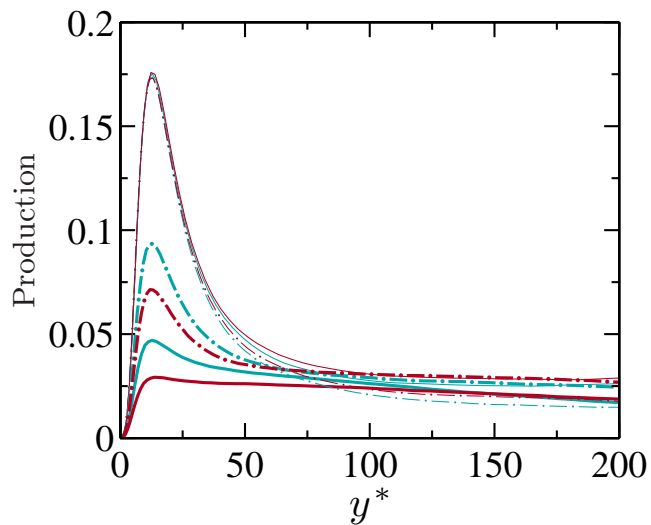

(b)

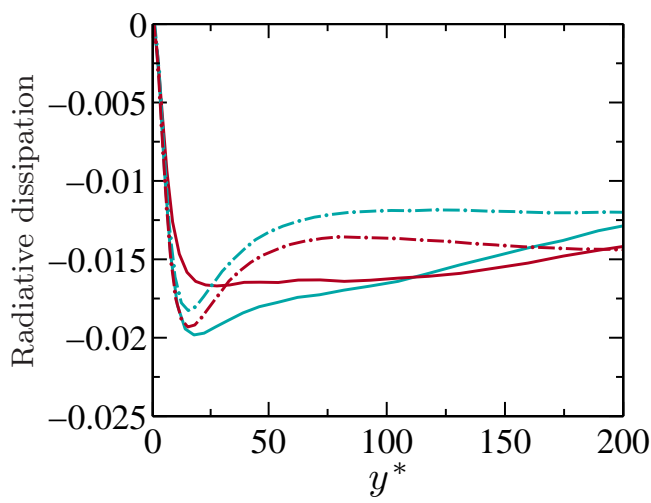

(d)

Figure 23. Profiles of enthalpy rms (a) and its production term (b), molecular dissipation (c) and radiative dissipation (d) using semi-local scaling on the cold side (blue color) and hot side (red color) in cases A (thin plain line), A_R (thick plain line), C (thin dashed-dotted line) and C_R (thick dashed-dotted line).

that is extensively used to relate the thermal eddy diffusivity $a_{t}$ to the turbulent eddy viscosity $\nu_{t}$. It is defined as

$$
\operatorname{Pr}_{t}=\frac{\nu_{t}}{a_{t}}=\frac{-\bar{\rho} \widetilde{u^{\prime \prime} v^{\prime \prime}}}{-\bar{\rho} v^{\prime \prime} \mathrm{h}^{\prime \prime} / \overline{c_{p}}} \frac{\mathrm{d} \bar{T} / \mathrm{d} Y}{\mathrm{~d} \bar{u} / \mathrm{d} Y} .
$$

This definition can alternatively be based on the gradient of Favre average of temperature and velocity. As mentioned earlier, the difference between Reynolds and Favre averaging approaches is small in the studied conditions. The turbulent Prandtl number concept can be erroneous for complex configurations. It is however valid for inhomogeneous turbulent flows in one directions such as turbulent boundary layers. Computational cost becoming tremendous for wall-resolved Large-Eddy Simulation (LES) as the Reynolds number increases (Piomelli 2008), wall models are necessary in order not to resolve the boundary inner layer. Thus, when considering heat transfer in wall-modeled LES (Benarafa et al. 2007; Larsson et al. 2011; Kawai \& Larsson 2012; Zhang et al. 2013b), the turbulent Prandtl number model, which is here a valid approach, is a primordial component of 




(a)



(c)

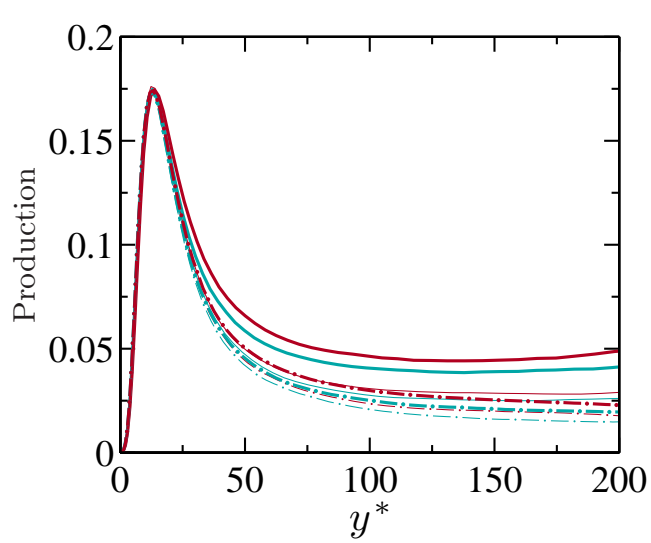

(b)



(d)

Figure 24. Profiles of enthalpy rms (a) and its production term (b), molecular dissipation (c) and radiative dissipation (d) using radiation-based scaling on the cold side (blue color) and hot side (red color) in cases A (thin plain line), A_R (thick plain line), C (thin dashed-dotted line) and C_R (thick dashed-dotted line).

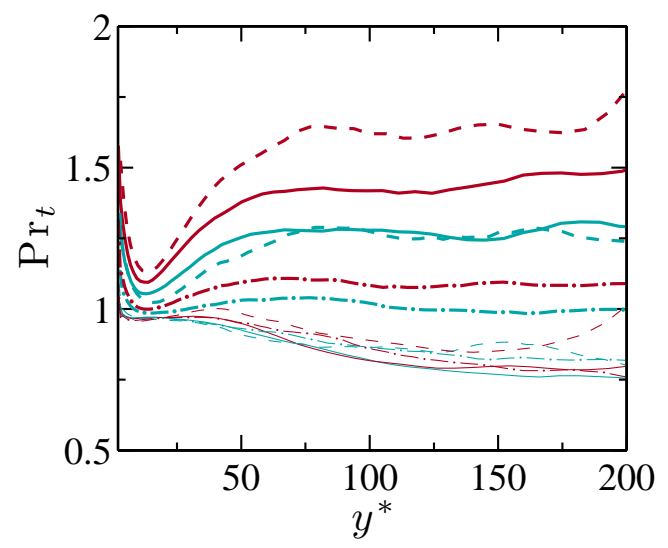

Figure 25. Profiles of the turbulent Prandtl number on the cold side (blue color) and hot side (red color) in cases A (thin plain line), A_R (thick plain line), B (thin dashed line), B_R (thick dashed line), C (thin dashed-dotted line) and C_R (thick dashed-dotted line). 


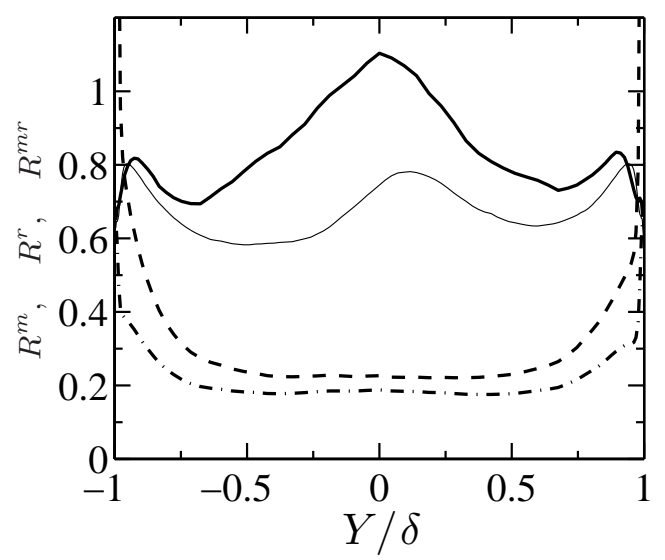

Figure 26. Profiles of time scale ratio $R^{m}$ in cases A (thin plain line) and A_R. In case A_R, different definitions of the thermal time scale are based on: Molecular dissipation only $\left(R^{m}\right.$ : thick plain line); Radiative dissipation only $\left(R^{r}\right.$ : thick dashed line); Both dissipation terms $\left(R^{m r}\right.$ : thick dashed-dotted line).



Figure 27. Comparison of predicted turbulent Prandtl number with DNS results in cases A and A_R (only the cold side is shown). Case A: DNS (filled circles); Model A ${ }^{m}$ (thin dotted line); Model B ${ }^{m}$ (thin dashed line). Case A_R: DNS (empty circles); Model B ${ }^{m}$ (thick dashed line); Model $\mathrm{A}^{m r}$ (thick dotted line); Model $\mathrm{B}^{m r}$ (thick dashed-dotted line).

the wall model. The DNS results are then here analyzed in terms of resulting turbulent Prandtl number in order to understand the impact on heat transfer wall modeling.

The profiles of $\operatorname{Pr}_{t}$ are presented in figure 25 for all studied cases. The profile of the turbulent Prandtl number in boundary layers has been widely studied (Kays 1994) and results for cases without radiation are consistent with channel flow simulations in the literature with air $(\mathrm{Pr}=0.71)$ and fixed temperature at the wall (Kim \& Moin 1987; Kong et al. 2000): The value is close to unity at the wall and decreases along the wallnormal distance, reaching an asymptotic value for high Reynolds number flows. Deviation of case B from cases A and $\mathrm{C}$ is attributed to mean density effects. Cases with radiation exhibit much stronger differences: The obtained profiles of turbulent Prandtl number are all above the standard ones without radiation. Profiles for cases A_R, B_R and C_R on the two sides of the channel are all different from each other but have the same pattern: $\operatorname{Pr}_{t}$ decreases from a value larger than unity at the wall until $y^{*} \approx 15$, then increases between $y^{*}=15$ and 75 to reach a plateau beyond. The value $y^{*}=75$ also corresponds to 
the value where the radiation-based turbulent heat flux reaches a plateau (see figures 15 and 22). This result is due to the fact that $\operatorname{Pr}_{t}$ is related to $\overline{\rho v^{\prime \prime} h^{\prime \prime}}{ }^{\dagger}$ :

$$
\operatorname{Pr}_{t} \propto \frac{\mathrm{d} \bar{T} / \mathrm{d} Y}{-\bar{\rho} \widetilde{v^{\prime \prime} \mathrm{h}^{\prime \prime}}} \approx \frac{1}{\bar{\lambda}} \frac{\left(1-\overline{\rho v^{\prime \prime} \mathrm{h}^{\prime \prime}}{ }^{\dagger}\right)}{-\overline{\rho v^{\prime \prime} \mathrm{h}^{\prime \prime}}{ }^{\dagger}}
$$

Finally, the effects of Reynolds number and wall temperature difference are consistent with previous results: Case C_R is closer to results without radiation while the hot wall profile in case B_R presents the largest difference.

Since radiation can modify the profile of the turbulent Prandtl number significantly, a dedicated model for $\mathrm{Pr}_{t}$ in wall-modeled LES is necessary. Obviously, considering a constant turbulent Prandtl number or algebraic functions of $\operatorname{Pr}_{t}$ from Kays (1994) only valid for equilibrium turbulent boundary layers is not appropriate since these approaches are not accounting for radiation. Four-equations closure models developed for Reynolds Averaged Numerical Simulations (RANS) are then first investigated because these more comprehensive models can calculate variable turbulent Prandtl numbers. These models usually use the time scale ratio $R=\tau_{t} / \tau_{u}$, where the velocity turbulent time scale $\tau_{u}$ and thermal turbulent time scale $\tau_{t}$ are defined as

$$
\tau_{u}=\frac{k}{\epsilon}, \quad \tau_{t}=\frac{\widetilde{\mathrm{h}^{\prime \prime} \mathrm{h}^{\prime \prime}}}{2 \epsilon_{h}},
$$

and $\epsilon$ and $\epsilon_{h}$ are dissipation terms of the turbulent kinetic energy $k$ and enthalpy variance, respectively. When radiation is accounted for, there are two types of dissipation, i.e. molecular and radiative dissipations, denoted here as $\epsilon_{h}^{m}$ and $\epsilon_{h}^{r}$ respectively. Therefore, three thermal time scales can be defined:

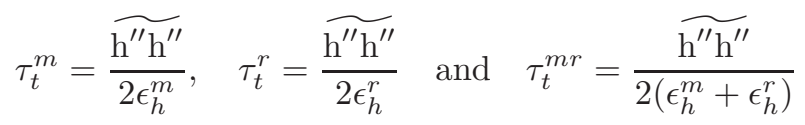

The first one is only based on molecular dissipation, the second one only on radiative dissipation and the third one on the sum of these two previous effects. The different corresponding time scale ratios $R^{m}=\tau_{t}^{m} / \tau_{u}, R^{r}=\tau_{t}^{r} / \tau_{u}$ and $R^{m r}=\tau_{t}^{m r} / \tau_{u}$ are shown in figure 26 in case $\mathrm{A}$ and A_R. The common time scale ratio $R^{m}$ based on thermal molecular dissipation is larger than the others in case A_R while the one based on radiative dissipation $R^{r}$ is much smaller. Finally, the combination of the two dissipative terms in the definition of $R^{m r}$ leads to the shortest thermal turbulent time scale. As radiation dissipation dominates, this latter definition is close to the one based on radiative dissipation only.

Different models for the thermal turbulent diffusivity $a_{t}$, which use the time scale ratio $R$ as input and lead to different expressions of the turbulent Prandtl number, are now considered. These models are completed with four transport equations for $k, \epsilon, \widehat{\mathrm{h}^{\prime \prime} \mathrm{h}^{\prime \prime}}$ and $\epsilon_{h}$ to compute the different turbulent time scales. Here, DNS data are used to assess their accuracy. The turbulent thermal diffusivity $a_{t}$ is modeled as

$$
a_{t}=C_{\lambda} k \tau_{m} f_{\lambda},
$$

where $\tau_{m}$ is a mixed time scale expressed from $\tau_{u}$ and $R, C_{\lambda}=0.1$ a model constant usually set to retrieve the asymptotically constant turbulent Prandtl $(\approx 0.85)$ in air at high Reynolds number and $f_{\lambda}$ is a damping function accounting for low-Reynolds effects at the immediate vicinity of the wall. Two formulations for $\tau_{m}$ are considered. The first 
one (referred as model A) used by Nagano \& Kim (1988) and Soufiani et al. (1990) is written as

$$
\tau_{m}=\tau_{u}(2 R)^{m} \quad \text { with } \quad m=0.5
$$

while the second formulation (referred as model B) from Abe et al. (1995) can is given by

$$
\tau_{m}=\tau_{u} \frac{2 R}{R+C_{m}} \quad \text { with } \quad C_{m}=0.5
$$

For each of these models, the thermal turbulent time scale and the corresponding ratio $R$ can be evaluated either only from the molecular dissipation (models $\mathrm{A}^{m}$ and $\mathrm{B}^{m}$ ) or from the total dissipation term, sum of molecular and radiative dissipative terms (models $\mathrm{A}^{m r}$ and $\mathrm{B}^{m r}$ ). The latter approach was already proposed by Ammouri et al. (1994). Models $\mathrm{A}^{m}, \mathrm{~A}^{m r}, \mathrm{~B}^{m}$ and $\mathrm{B}^{m r}$ are a priori tested using DNS profiles of time scale ratios $R^{m}$ and $R^{m r}$ to compute $a_{t}$. Damping functions $f_{\lambda}$ for $\mathrm{A}^{m}$ and $\mathrm{A}^{m r}$ are taken from Soufiani et al. (1990), and for models $\mathrm{B}^{m}$ and $\mathrm{B}^{m r}$ from Abe et al. (1995). The predicted profiles of $a_{t}$ are combined with the turbulent viscosity profiles extracted from DNS results to compute profiles of turbulent Prandtl number $\operatorname{Pr}_{t}$ that are presented in figure 27 .

In case $\mathrm{A}$, without radiation, predictions of models $\mathrm{A}^{m}$ and $\mathrm{B}^{m}$ are similar and tend to an asymptotic value of $\mathrm{Pr}_{t}$. Agreement with the DNS data is correct although the turbulent Prandtl number obtained in DNS does not reach a plateau and keeps decreasing. This behavior can be attributed to low-Reynolds effects in the core of the channel but also to the specific configuration of different wall temperatures, which leads to an increase of enthalpy fluctuations, starting here at $y^{+} \approx 100$, in the center of the channel (see figure 4).

With radiation, results of model $\mathrm{B}^{m}$ (and $\mathrm{A}^{m}$, not presented here) show that, if only the molecular dissipation is accounted for, the turbulent Prandtl number is significantly underestimated and that a wrong trend is predicted. On the other hand, when both molecular and radiative dissipations are accounted for in the definition of the thermal time scale, the turbulent Prandtl number predicted by models $\mathrm{A}^{m r}$ and $\mathrm{B}^{m r}$ is correctly enhanced by radiation effects but the discrepancies with the DNS profile of case A_R remain important.

Using previous results with the radiation-based scaling, another model for $\mathrm{Pr}_{t}$ is proposed to account for radiation effects in turbulent boundary layers. In section 3.2, it has been demonstrated that the non-dimensional turbulent heat flux, when normalized with radiation-based scaling, agree well between different cases with and without radiation, i.e.,

$$
{\overline{\rho v^{\prime \prime} \mathrm{h}_{R}^{\prime \prime}}}_{R}^{\dagger} \approx{\overline{\rho v^{\prime \prime} \mathrm{h}^{\prime \prime}}}_{0}^{\dagger},
$$

where subscript indices $R$ and 0 correspond to quantities in cases with and without radiation, respectively. For cases with radiation, the thermal turbulent diffusivity can then be written

$$
a_{t, R}=\frac{1}{\bar{\rho}_{R}{\overline{c_{p}}}_{R}} \frac{\overline{\rho v^{\prime \prime} \mathrm{h}_{R}^{\prime \prime}}}{\mathrm{d} \bar{T}_{R} / \mathrm{d} \bar{y}} \approx \frac{1}{\bar{\rho}_{R}{\overline{c_{p}}}_{R}} \frac{\overline{\mid q_{R}^{\dagger}}(Y) \mid}{\overline{\mathrm{d}} \bar{T}_{R} / \mathrm{d} \bar{y}} \overline{\rho v^{\prime \prime} \mathrm{h}^{\prime \prime}}{ }_{0}^{\dagger}
$$

For cases without radiation, $\overline{\rho v^{\prime \prime} \mathrm{h}^{\prime \prime}}{ }_{0}^{\dagger}$ is expressed as

$$
\overline{\rho v^{\prime \prime} \mathrm{h}_{0}^{\prime \prime}}{ }_{0}^{\dagger}=\frac{\overline{\rho v^{\prime \prime} \mathrm{h}_{0}^{\prime \prime}}}{\overline{\left|q_{w, 0}^{c d}\right|}}=\frac{a_{t, 0}}{\overline{a_{0}}+a_{t, 0}} \frac{\mathrm{d} \bar{T}_{0} / \mathrm{d} \bar{y}}{\left|\mathrm{~d} \bar{T}_{0} / \mathrm{d} \bar{y}\right|}=\frac{\nu_{t, 0}}{\overline{a_{0}} \operatorname{Pr}_{t}^{0}+\nu_{t, 0}} \frac{\mathrm{d} \bar{T}_{0} / \mathrm{d} \bar{y}}{\left|\mathrm{~d} \bar{T}_{0} / \mathrm{d} \bar{y}\right|},
$$

where $a_{0}$ is the thermal diffusivity. From equations (3.41) and (3.42), the turbulent 


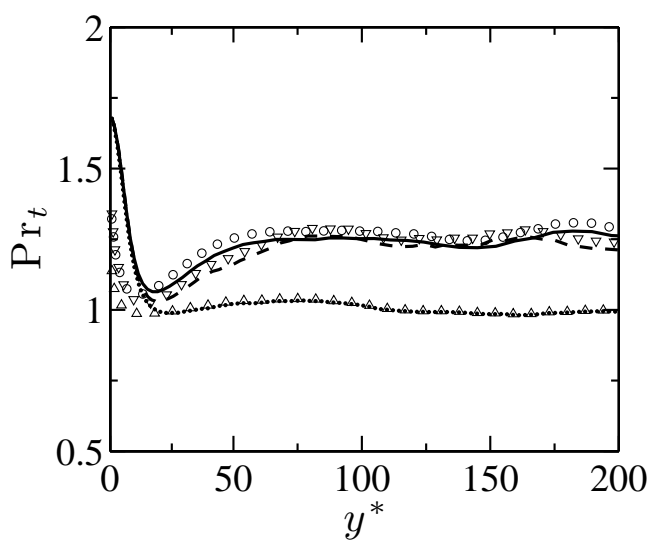

(a)

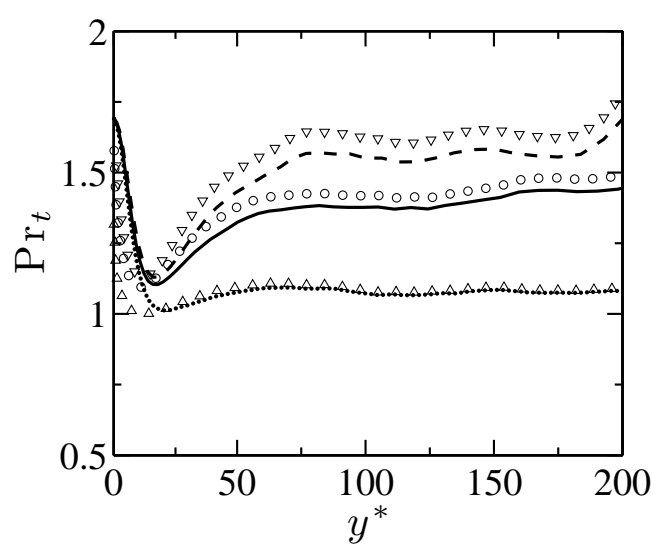

(b)

Figure 28. Profiles of modeled turbulent Prandtl number on the cold side (a) and hot side (b) compared to DNS results in cases A_R (DNS: Circles; Model: Plain line) , B_R (DNS: Down-pointing triangles; Model: Dashed line) and C_R (DNS: Up-pointing triangles; Model: Dotted line).

Prandtl number $\operatorname{Pr}_{t, R}$ that accounts for radiation effects can be calculated as

$$
\operatorname{Pr}_{t, R}=\frac{\nu_{t, R}}{a_{t, R}}=\bar{\rho}_{R}{\overline{c_{p}}}_{R} \frac{\left|\mathrm{d} \bar{T}_{R} / \mathrm{d} \bar{y}\right|}{\left|\overline{q_{R}^{\dagger}}(Y)\right|} \frac{\nu_{t, R}\left(\overline{a_{0}} \operatorname{Pr}_{t}^{0}+\nu_{t, 0}\right)}{\nu_{t, 0}} .
$$

Neglecting radiation effects on the velocity field, $\nu_{t, 0}$ can then be replaced with $\nu_{t, R}$. Assuming also that $a_{R} \approx a_{0}$ finally gives

$$
\operatorname{Pr}_{t, R}=\bar{\rho}_{R}{\overline{c_{p}}}_{R} \frac{\left|\mathrm{d} \bar{T}_{R} / \mathrm{d} \bar{y}\right|}{\left|\overline{q_{R}^{\dagger}}(Y)\right|}\left(\overline{a_{R}} \operatorname{Pr}_{t}^{0}+\nu_{t, R}\right),
$$

Effects of radiation are included through $\overline{q_{R}^{\dagger}}(Y)$ which is related to the radiative flux and through the change of the mean temperature profile in the term $\left|\mathrm{d} \bar{T}_{R} / \mathrm{d} \bar{y}\right|$. When no radiative energy transfer is considered, the right-hand-side of equation (3.44) simplifies to $\operatorname{Pr}_{t}^{0}$, profile of turbulent Prandtl number in cases without radiation. This last piece of the model can either be given by an algebraic formula or by a more complex model. Here, the formula proposed by Kays (1994) is considered:

$$
\operatorname{Pr}_{t}^{0}=\frac{1}{0.5882+0.228\left(\nu_{t, R} / \overline{\nu_{R}}\right)-0.0441\left(\nu_{t, R} / \overline{\nu_{R}}\right)^{2}\left[1-\exp \left(\frac{-5.165}{\nu_{t, R} / \overline{\nu_{R}}}\right)\right]} .
$$

Equations (3.44) and (3.45) are the base of a turbulent Prandtl model accounting for radiation, to be applied in turbulent boundary layers: It can be used in RANS simulations but also in wall-model for large eddy simulations. Using DNS data to evaluate equations (3.44) and (3.45), results of the proposed model are compared with DNS results in figure 28 for all studied cases. The agreement of the model with DNS is impressive, showing the importance of the radiation-based scaling. The validity of the model is nonetheless limited to equilibrium boundary layers since it is the scope of the investigated cases and of equation (3.45). 


\section{Conclusion}

Direct numerical simulations of turbulent channel flows of burnt gases composition under different conditions (Reynolds number, wall temperature difference) are analyzed with and without accounting for radiative energy transfer, computed with a Monte-Carlo method. In addition to the modification of the mean temperature profiles and associated wall laws, fluctuations of temperature and turbulent heat transfer are significantly reduced by radiation in the studied conditions for two main reasons. On the one hand, radiation indirectly influences production terms that appear in the balance equation of these quantities by changing gradients of the mean temperature field. On the other hand, the additional radiative power source term in the energy balance equation generates new terms in transport equations of enthalpy variance and turbulent heat flux. The two new terms, correlations between radiative power and enthalpy fluctuations and between radiative power and wall-normal velocity fluctuations, appear as loss terms in these equations. There is therefore a strong interaction between the fluctuations of radiative power and temperature. The latter then influences the mean temperature field through turbulent transport. However, the widely studied impact of turbulent fluctuations on the mean radiative power is negligible in the considered channel flows where no combustion takes place.

The different types of impact of radiation on the turbulent heat flux, the enthalpy variance and their transport equation terms are shown to be different in each case, for different wall temperature and different Reynolds numbers. The usual collapsing of wall-scaled variable profiles under these different conditions is then not retrieved when radiation is taken into account. This effect is corrected by introducing a radiation-based scaling in order to correctly quantify the balance between molecular diffusion and turbulent transport. Very good agreements between profiles of scaled turbulent heat flux and other related variables such as production terms are obtained for all studied cases. The balance between scaled molecular dissipation (or the enthalpy-pressure-gradient term) and the radiation loss term, that both compensate production outside of the boundary buffer layer, is determined by the magnitude of radiative energy transfer.

Finally, using radiation-based scaling, a model for the turbulent Prandtl number in boundary layers with radiation effects is proposed. A priori validation shows that profiles of turbulent Prandtl number that are significantly different from the cases without radiation are accurately predicted. This model can be used in RANS or wall-modeled LES when radiation is able to modify the boundary layer structure.

\section{Acknowledgements}

We thank the China Scholarship Council (CSC) for granting a doctoral fellowship to Yufang Zhang. This work was granted access to the HPC resources of [CCRT/CINES/IDRIS] under the allocations 2012-2b0164 and 2013-2b0164 made by GENCI (Grand Equipement National de Calcul Intensif). Vincent Moureau and Ghislain Lartigue from the CORIA lab, and the SUCCESS scientific group are acknowledged for providing the YALES2 code.

\section{Appendix A. Derivation of transport equations for enthalpy root-mean-square and turbulent heat flux}

In a low Mach-number formulation, the pressure in the enthalpy equation (2.3) is the thermodynamic pressure which is constant in the studied cases. The enthalpy equation 
can then be simplified to

$$
\frac{\partial(\rho \mathrm{h})}{\partial t}+\frac{\partial\left(\rho u_{j} \mathrm{~h}\right)}{\partial x_{j}}=-\frac{\partial q_{j}^{c d}}{\partial x_{j}}+P^{R}
$$

Averaging equation (A 1) makes the turbulent heat flux appear on the right-hand-side and gives

$$
\frac{\partial}{\partial t}(\bar{\rho} \widetilde{\mathrm{h}})+\frac{\partial}{\partial x_{j}}\left(\bar{\rho} \widetilde{u_{j}} \widetilde{\mathrm{h}}\right)=-\frac{\partial \overline{q_{j}^{c d}}}{\partial x_{j}}+\overline{P^{R}}-\frac{\partial}{\partial x_{j}}\left(\bar{\rho} \widetilde{u_{j}^{\prime \prime} \mathrm{h}^{\prime \prime}}\right) .
$$

A transport equation for $\widetilde{\mathrm{h}}^{2} / 2$ is obtained by multiplying equation (A 2) by $\widetilde{\mathrm{h}}$ :

$$
\frac{\partial}{\partial t}\left(\bar{\rho} \frac{\widetilde{\mathrm{h}}^{2}}{2}\right)+\frac{\partial}{\partial x_{j}}\left(\bar{\rho} \widetilde{u_{j}} \frac{\widetilde{\mathrm{h}}^{2}}{2}\right)=-\widetilde{\mathrm{h}} \frac{\partial \overline{q_{j}^{c d}}}{\partial x_{j}}+\widetilde{\mathrm{h}} \overline{P^{R}}-\widetilde{\mathrm{h}} \frac{\partial}{\partial x_{j}}\left(\bar{\rho} \widetilde{u_{j}^{\prime \prime} \mathrm{h}^{\prime \prime}}\right) .
$$

Similarly, a transport equation for $\widetilde{\mathrm{h}^{2}} / 2$ is obtained by multiplying equation (A 1 ) by $\mathrm{h}$ and averaging, which gives

$$
\frac{\partial}{\partial t}\left(\bar{\rho} \frac{\widetilde{\mathrm{h}^{2}}}{2}\right)+\frac{\partial}{\partial x_{j}}\left(\bar{\rho} u_{j} \frac{\mathrm{h}^{2}}{2}\right)=-\overline{\mathrm{h}} \overline{\frac{\partial q_{j}^{c d}}{\partial x_{j}}}+\overline{\mathrm{h} P^{R}}
$$

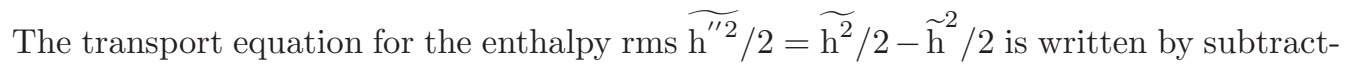
ing equation (A 3) from equation (A 4):

$\frac{\partial}{\partial t}\left(\bar{\rho} \frac{\widetilde{\mathrm{h}^{\prime \prime 2}}}{2}\right)+\frac{\partial}{\partial x_{j}}\left(\bar{\rho}\left(\widetilde{u_{j} \frac{\mathrm{h}^{2}}{2}}-\widetilde{u_{j}} \frac{\widetilde{\mathrm{h}}^{2}}{2}\right)\right)=-\overline{\mathrm{h} \frac{\partial q_{j}^{c d}}{\partial x_{j}}}+\widetilde{\mathrm{h}} \frac{\partial \overline{q_{j}^{c d}}}{\partial x_{j}}+\overline{\mathrm{h} P^{R}}-\widetilde{\mathrm{h}} \overline{P^{R}}+\widetilde{\mathrm{h}} \frac{\partial}{\partial x_{j}}\left(\bar{\rho} \widetilde{u_{j}^{\prime \prime} \mathrm{h}^{\prime \prime}}\right)$.

The second term on the left-hand-side can be written as

$$
\widetilde{u_{j} \frac{\mathrm{h}^{2}}{2}}-\widetilde{u_{j}} \frac{\widetilde{\mathrm{h}}^{2}}{2}=\frac{1}{2} \widetilde{u_{j} \mathrm{~h}^{\prime \prime 2}}+\widetilde{\mathrm{h}} \widetilde{u_{j}^{\prime \prime} \mathrm{h}^{\prime \prime}}+\frac{1}{2} \widetilde{u_{j}^{\prime \prime} \mathrm{h}^{\prime \prime} \mathrm{h}^{\prime \prime}} .
$$

Inserting this expression in equation (A 5 ) gives

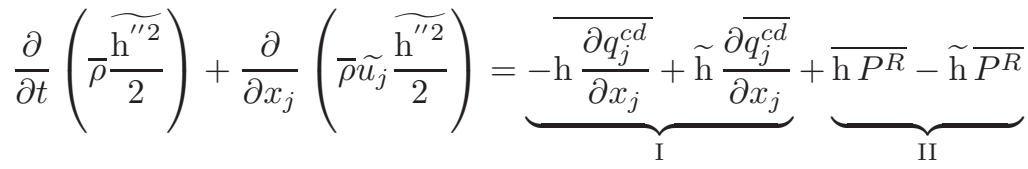

$$
\begin{aligned}
& +\underbrace{\widetilde{\mathrm{h}} \frac{\partial}{\partial x_{j}}\left(\bar{\rho} \widetilde{u_{j}^{\prime \prime} \mathrm{h}^{\prime \prime}}\right)-\frac{\partial}{\partial x_{j}}\left(\tilde{\mathrm{h}} \bar{\rho} \widetilde{u_{j}^{\prime \prime} \mathrm{h}^{\prime \prime}}\right)}_{\text {III }} \\
& -\frac{1}{2} \frac{\partial}{\partial x_{j}}\left(\bar{\rho} \widetilde{u_{j}^{\prime \prime} \mathrm{h}^{\prime \prime} \mathrm{h}^{\prime \prime}}\right) .
\end{aligned}
$$

With the following simplifications on terms I, II and III,

$$
\begin{aligned}
\mathrm{I}:-\overline{\mathrm{h} \frac{\partial q_{j}^{c d}}{\partial x_{j}}}+\widetilde{\mathrm{h}} \frac{\partial \overline{q_{j}^{c d}}}{\partial x_{j}} & =-\overline{\left(\widetilde{\mathrm{h}}+\mathrm{h}^{\prime \prime}\right) \frac{\partial q_{j}^{c d}}{\partial x_{j}}}+\widetilde{\mathrm{h}} \frac{\partial \overline{q_{j}^{c d}}}{\partial x_{j}} \\
& =-\overline{\mathrm{h}^{\prime \prime} \frac{\partial q_{j}^{c d}}{\partial x_{j}}}=-\overline{\mathrm{h}^{\prime \prime}} \frac{\partial \overline{q_{j}^{c d}}}{\partial x_{j}}-\overline{\mathrm{h}^{\prime \prime} \frac{\partial q_{j}^{c d^{\prime}}}{\partial x_{j}}}
\end{aligned}
$$




$$
\begin{aligned}
& \text { II : } \overline{\mathrm{h} P^{R}}-\widetilde{\mathrm{h}} \overline{P^{R}}=\overline{\left(\widetilde{\mathrm{h}}+\mathrm{h}^{\prime \prime}\right) P^{R}}-\widetilde{\mathrm{h}} \overline{P^{R}}=\overline{\mathrm{h}^{\prime \prime} P^{R}}=\overline{\mathrm{h}^{\prime \prime}} \overline{P^{R}}+\overline{\mathrm{h}^{\prime \prime} P^{R^{\prime}}} \text {, } \\
& \text { III : } \quad \widetilde{\mathrm{h}} \frac{\partial}{\partial x_{j}}\left(\widetilde{\rho} \widetilde{u}_{j}^{\prime \prime \mathrm{h}^{\prime \prime}}\right)-\frac{\partial}{\partial x_{j}}\left(\widetilde{\mathrm{h}} \bar{\rho} \widetilde{u_{j}^{\prime \prime} \mathrm{h}^{\prime \prime}}\right)=-\bar{\rho} \widetilde{u_{j}^{\prime \prime} \mathrm{h}^{\prime \prime}} \frac{\partial \widetilde{\mathrm{h}}}{\partial x_{j}},
\end{aligned}
$$

equation (A 7) becomes

$$
\begin{aligned}
\frac{\partial}{\partial t}\left(\bar{\rho} \frac{\widetilde{\mathrm{h}^{\prime \prime 2}}}{2}\right)+\frac{\partial}{\partial x_{j}}\left(\bar{\rho} \widetilde{u_{j}} \frac{\widetilde{\mathrm{h}^{\prime \prime 2}}}{2}\right)=-\widetilde{\mathrm{h}^{\prime \prime} \frac{\partial q_{j}^{c d^{\prime}}}{\partial x_{j}}}+\overline{\mathrm{h}^{\prime \prime} P^{R^{\prime}}}-\bar{\rho} \widetilde{u_{j}^{\prime \prime} \mathrm{h}^{\prime \prime}} \frac{\partial \widetilde{\mathrm{h}}}{\partial x_{j}} \\
-\frac{1}{2} \frac{\partial}{\partial x_{j}}\left(\bar{\rho} \widetilde{u_{j}^{\prime \prime} \mathrm{h}^{\prime \prime} \mathrm{h}^{\prime \prime}}\right)+\overline{\mathrm{h}^{\prime \prime}}\left(-\frac{\partial \overline{q_{j}^{c d}}}{\partial x_{j}}+\overline{P^{R}}\right) .
\end{aligned}
$$

For any quantities $\psi$ and $\phi$, cross-correlations between Favre and Reynolds fluctuations have the following properties: $\overline{\phi^{\prime} \psi^{\prime \prime}}=\overline{\phi^{\prime} \psi^{\prime}}$ and $\widehat{\phi^{\prime} \psi^{\prime \prime}}=\widehat{\phi^{\prime \prime} \psi^{\prime \prime}}$. The first term on the right-hand-side is written as

$$
-\overline{-\mathrm{h}^{\prime \prime} \frac{\partial q_{j}^{c d^{\prime}}}{\partial x_{j}}}=-\overline{\mathrm{h}^{\prime} \frac{\partial q_{j}^{c d^{\prime}}}{\partial x_{j}}}=-\frac{\partial}{\partial x_{j}}\left(\overline{q_{j}^{c d^{\prime}} \mathrm{h}^{\prime}}\right)+\overline{q_{j}^{c d^{\prime}} \frac{\partial \mathrm{h}^{\prime}}{\partial x_{j}}}
$$

Only assuming constant thermodynamic pressure, the general transport equation for the enthalpy $\mathrm{rms}$ is therefore given by

$$
\begin{aligned}
& \frac{\partial}{\partial t}\left(\bar{\rho} \frac{\widetilde{\mathrm{h}^{\prime \prime 2}}}{2}\right)+\frac{\partial}{\partial x_{j}}\left(\bar{\rho} \widetilde{u_{j}} \frac{\widetilde{\mathrm{h}^{\prime \prime 2}}}{2}\right)=-\frac{\partial}{\partial x_{j}}\left(\overline{q_{j}^{c d^{\prime}} \mathrm{h}^{\prime}}\right)+\widetilde{q_{j}^{c d^{\prime}} \frac{\partial \mathrm{h}^{\prime}}{\partial x_{j}}}+\widetilde{\mathrm{h}^{\prime \prime} P^{R^{\prime}}}-\bar{\rho} \widetilde{u_{j}^{\prime \prime} \mathrm{h}^{\prime \prime}} \frac{\partial \widetilde{\mathrm{h}}}{\partial x_{j}} \\
& -\frac{1}{2} \frac{\partial}{\partial x_{j}}\left(\bar{\rho} \widetilde{u_{j}^{\prime \prime} \mathrm{h}^{\prime \prime} \mathrm{h}^{\prime \prime}}\right)+\overline{\mathrm{h}^{\prime \prime}}\left(-\frac{\partial \overline{q_{j}^{c d}}}{\partial x_{j}}+\overline{P^{R}}\right) .
\end{aligned}
$$

In the investigated channel flow configuration, averaged quantities do not depend on the homogeneous directions $\mathrm{X}$ and $\mathrm{Z}$ and the left-hand-side is null. The transport equation therefore becomes

$0=-\frac{\partial}{\partial y}\left(\overline{q_{y}^{c d^{\prime}} \mathrm{h}^{\prime}}\right)+\overline{q_{j}^{c d^{\prime}} \frac{\partial \mathrm{h}^{\prime}}{\partial x_{j}}}+\overline{\mathrm{h}^{\prime \prime} P^{R^{\prime}}}-\bar{\rho} \widetilde{v^{\prime \prime} \mathrm{h}^{\prime \prime}} \frac{\partial \widetilde{\mathrm{h}}}{\partial y}-\frac{1}{2} \frac{\partial}{\partial y}\left(\bar{\rho} \widetilde{v^{\prime \prime} \mathrm{h}^{\prime \prime} \mathrm{h}^{\prime \prime}}\right)+\overline{\mathrm{h}^{\prime \prime}}\left(-\frac{\partial \overline{q_{y}^{c d}}}{\partial y}+\overline{P^{R}}\right)$.

In the channel flow configuration, equation (A 2) gives

$$
-\frac{\partial \overline{q_{y}^{c d}}}{\partial y}+\overline{P^{R}}=\frac{\partial}{\partial y}\left(\widetilde{\rho} \widetilde{v^{\prime \prime} \mathrm{h}^{\prime \prime}}\right) .
$$

Modifying accordingly the last term in equation (A 14) finally gives equation (3.4) given in section 3.1.2:

$$
-\frac{\partial}{\partial y}\left(\overline{q_{y}^{c d^{\prime} \mathrm{h}^{\prime}}}\right)-\frac{1}{2} \frac{\partial}{\partial y}\left(\widetilde{\rho v^{\prime \prime} \mathrm{h}^{\prime \prime} \mathrm{h}^{\prime \prime}}\right)-\widetilde{\rho} \widetilde{v^{\prime \prime} \mathrm{h}^{\prime \prime}} \frac{\partial \widetilde{\mathrm{h}}}{\partial y}+\widetilde{q_{i}^{c d^{\prime}} \frac{\partial \mathrm{h}^{\prime}}{\partial x_{i}}}+\widetilde{\mathrm{h}^{\prime \prime} P^{R^{\prime}}}+\widetilde{\mathrm{h}^{\prime \prime}} \frac{\partial}{\partial y}\left(\bar{\rho} \widetilde{v^{\prime \prime} \mathrm{h}^{\prime \prime}}\right)=0
$$

The transport equation for the quantity $\widetilde{v^{\prime \prime} h^{\prime \prime}}$ is obtained similarly by subtracting the one for $\widetilde{v} \widetilde{\mathrm{h}}$ from the transport equation for $\widetilde{v \mathrm{~h}}$. The transport equation of $\widetilde{v}$ is given by

$$
\frac{\partial}{\partial t}(\bar{\rho} \widetilde{v})+\frac{\partial}{\partial x_{j}}\left(\bar{\rho} \widetilde{u_{j}} \widetilde{v}\right)=-\frac{\partial \bar{p}}{\partial y}+\frac{\partial \overline{\tau_{2 j}}}{\partial x_{j}}-\frac{\partial}{\partial x_{j}}\left(\bar{\rho} \widetilde{u_{j}^{\prime \prime} v^{\prime \prime}}\right) .
$$


By multiplying equation (A 15 ) by $\widetilde{\mathrm{h}}$, equation (A 2) by $\widetilde{v}$ and summing them, a transport equation for $\widetilde{v} \widetilde{h}$ is obtained:

$\frac{\partial}{\partial t}(\bar{\rho} \widetilde{v} \widetilde{\mathrm{h}})+\frac{\partial}{\partial x_{j}}\left(\bar{\rho} \widetilde{u_{j}} \widetilde{v} \tilde{\mathrm{h}}\right)=-\widetilde{\mathrm{h}} \frac{\partial \bar{p}}{\partial y}+\widetilde{\mathrm{h}} \frac{\partial \overline{\tau_{2 j}}}{\partial x_{j}}-\widetilde{v} \frac{\partial \overline{q_{j}^{c d}}}{\partial x_{j}}+\widetilde{v} \overline{P^{R}}-\widetilde{\mathrm{h}} \frac{\partial}{\partial x_{j}}\left(\bar{\rho} \widetilde{u_{j}^{\prime \prime} v^{\prime \prime}}\right)-\widetilde{v} \frac{\partial}{\partial x_{j}}\left(\bar{\rho} \widetilde{u_{j}^{\prime \prime} \mathrm{h}^{\prime \prime}}\right)$.

Similarly, multiplying the instantaneous transport equation for $v$ by h, multiplying the one for h by $v$ and averaging the sum gives

$$
\frac{\partial}{\partial t}(\bar{\rho} \widetilde{\mathrm{h}})+\frac{\partial}{\partial x_{j}}\left(\widetilde{\rho} \widetilde{u_{j} v \mathrm{~h}}\right)=-\overline{\mathrm{h} \frac{\partial p}{\partial y}}+\overline{\mathrm{h}} \overline{\frac{\partial \tau_{2 j}}{\partial x_{j}}}-\overline{v \frac{\partial q_{j}^{c d}}{\partial x_{j}}}+\overline{v P^{R}}
$$

where

$$
\widetilde{u_{j} v \mathrm{~h}}=\widetilde{u_{j}} \widetilde{v \mathrm{~h}}+\widetilde{\mathrm{h}} \widetilde{u_{j}^{\prime \prime} v^{\prime \prime}}+\widetilde{v} \widetilde{u_{j}^{\prime \prime} \mathrm{h}}+\widetilde{u_{j}^{\prime \prime} v^{\prime \prime} \mathrm{h}^{\prime \prime}} .
$$

The transport equation for $\widetilde{v h}$ can therefore be written as

$$
\begin{aligned}
\frac{\partial}{\partial t}(\bar{\rho} \widetilde{\mathrm{h}})+\frac{\partial}{\partial x_{j}}\left(\bar{\rho} \widetilde{u_{j}} \widetilde{v \mathrm{~h}}\right)=-\overline{\mathrm{h} \frac{\partial p}{\partial y}}+\overline{\mathrm{h} \frac{\partial \tau_{2 j}}{\partial x_{j}}}-\overline{v \frac{\partial q_{j}^{c d}}{\partial x_{j}}}+\overline{v P^{R}} \\
-\frac{\partial}{\partial x_{j}}\left(\bar{\rho} u_{j}^{\prime \prime} v^{\prime \prime} \mathrm{h}^{\prime \prime}\right)-\frac{\partial}{\partial x_{j}}\left(\bar{\rho} \widetilde{u_{j}^{\prime \prime} v^{\prime \prime}} \widetilde{\mathrm{h}}\right) \frac{\partial}{\partial x_{j}}-\left(\bar{\rho} \widetilde{u_{j}^{\prime \prime} \mathrm{h}^{\prime \prime}} \widetilde{v}\right) .
\end{aligned}
$$

Subtracting equation (A 17) from equation (A 19) and carrying out simplifications similar to equations (A 8), (A 9) and (A 10) finally gives the following general transport equation for the turbulent heat flux:

$$
\begin{array}{r}
\frac{\partial}{\partial t}\left(\widetilde{\rho} \widetilde{v^{\prime \prime} \mathrm{h}^{\prime \prime}}\right)+\frac{\partial}{\partial x_{j}}\left(\bar{\rho} \widetilde{u_{j}} \widetilde{v^{\prime \prime} \mathrm{h}^{\prime \prime}}\right)=-\overline{\mathrm{h}^{\prime} \frac{\partial p^{\prime}}{\partial y}}+\frac{\partial}{\partial x_{j}}\left(\tau_{2 j}^{\prime} \mathrm{h}^{\prime}\right)-\widetilde{\tau_{2 j}^{\prime} \frac{\partial \mathrm{h}^{\prime}}{\partial x_{j}}} \\
-\frac{\partial}{\partial x_{j}}\left(\overline{q_{j}^{c d^{\prime}} v^{\prime}}\right)+\widetilde{q_{j}^{c d^{\prime}}} \frac{\partial v^{\prime}}{\partial x_{j}}+\overline{v^{\prime \prime} P^{R^{\prime}}}-\bar{\rho} \widetilde{u_{j}^{\prime \prime} v^{\prime \prime}} \frac{\partial \widetilde{\mathrm{h}}}{\partial x_{j}}-\bar{\rho} \widetilde{u_{j}^{\prime \prime} \mathrm{h}^{\prime \prime}} \frac{\partial \widetilde{v}}{\partial x_{j}} \\
-\frac{\partial}{\partial x_{j}}\left(\bar{\rho} \widetilde{u_{j}^{\prime \prime} v^{\prime \prime} \mathrm{h}^{\prime \prime}}\right)+\overline{\mathrm{h}^{\prime \prime}}\left(-\frac{\partial \bar{p}}{\partial y}+\frac{\partial \overline{\tau_{2 j}}}{\partial x_{j}}\right)+\overline{v^{\prime \prime}}\left(\overline{P^{R}}-\frac{\partial \overline{q_{j}^{c d}}}{\partial x_{j}}\right)
\end{array}
$$

In the channel flow configuration, the left-hand-side is null, averaged quantities only depend on the $\mathrm{Y}$ direction and $\tilde{v}=0$. Consequently, equation (A 20) can be simplified to equation (3.8) used in section 3.1.3:



\section{Appendix B. Level of temperature fluctuations in thermal boundary layers}

In a turbulent thermal boundary layer, the peak of root-mean-square temperature is located in the buffer layer. The peak value and position have been shown to depend on both Reynolds number and Prandtl number (Kim \& Moin 1987; Kawamura et al. 1998, 
1999; Abe et al. 2004). Hence, the maximum temperature rms is

$$
T_{\mathrm{rms}}^{\max }=T_{\mathrm{rms}}\left(y_{\mathrm{BL}}^{+}(\operatorname{Re}, \operatorname{Pr})\right)=T_{\mathrm{rms}}^{+, \max }(\operatorname{Re}, \operatorname{Pr}) T_{\tau},
$$

where $y_{\mathrm{BL}}^{+}$is the position of the peak and $T_{\mathrm{rms}}^{+, \max }$ is the maximum temperature rms, both in wall units. In air with $\mathrm{Pr}=0.71$, optimum value of temperature rms in wall units and its position become Reynolds-independent at sufficiently high Reynolds number (Kasagi et al. 1992; Kawamura et al. 1998, 1999; Kong et al. 2000; Abe et al. 2004). For air, it is found that $y_{\mathrm{BL}}^{+} \approx 12$ and $T_{\mathrm{rms}}^{+, \max } \approx 2.5$.

Common approximations of the friction coefficient and Nusselt number in ducts are

$$
c_{f}=\frac{\bar{\tau}_{w}}{\rho u_{b}^{2}} \approx \alpha \operatorname{Re}^{-0.2}, \mathrm{Nu}=\frac{\left|\overline{q_{w}^{c d}}\right| D_{h}}{\left|T_{w}-T_{b}\right| \lambda} \approx \beta \operatorname{Re}^{0.8} \operatorname{Pr}^{0.5}
$$

where $u_{b}$ is the bulk velocity, $T_{b}$ the bulk temperature, $D_{h}$ the hydraulic diameter of the duct and $\operatorname{Re}=\rho u_{b} D_{h} / \mu$. The constant coefficients are $\alpha=0.023$ and $\beta=0.022$ in air. The friction temperature is defined as $T_{\tau}=\left|\overline{q_{w}^{c d}}\right| /\left(\rho c_{p} u_{\tau}\right)$ where

$$
u_{\tau}=\left(\frac{\bar{\tau}_{w}}{\rho}\right)^{1 / 2}=u_{b} c_{f}^{1 / 2} \text { and }\left|\overline{q_{w}^{c d}}\right|=\frac{\mathrm{Nu}\left|T_{w}-T_{b}\right| \lambda}{D_{h}} .
$$

Introducing the friction coefficient and Nusselt number formulas in equation (B 2), the friction temperature is written as

$$
T_{\tau}=\alpha^{-0.5} \beta \operatorname{Re}^{-0.1} \operatorname{Pr}^{-0.5}\left|T_{w}-T_{b}\right| .
$$

As the friction velocity $u_{\tau}$ increases faster with the Reynolds number than the conductive heat flux, the friction temperature decreases with the Reynolds number and so is the maximum temperature rms. The relative variation of temperature is

$$
\frac{T_{\mathrm{rms}}^{\max }}{\bar{T}\left(y_{\mathrm{BL}}^{+}\right)}=\frac{T_{\mathrm{rms}}^{+, \max }(\mathrm{Re}, \operatorname{Pr})}{\operatorname{sign}\left(T_{b}-T_{w}\right) \bar{T}^{+}\left(y_{\mathrm{BL}}^{+}(\operatorname{Re}, \operatorname{Pr})\right)+\frac{T_{w}}{T_{\tau}}} .
$$

Introducing equation (B 4), it is found that

$$
\frac{T_{\mathrm{rms}}^{\max }}{\bar{T}\left(y_{\mathrm{BL}}^{+}\right)}=\frac{T_{\mathrm{rms}}^{+, \max }(\operatorname{Re}, \operatorname{Pr})}{\operatorname{sign}\left(T_{b} / T_{w}-1\right) \bar{T}^{+}\left(y_{\mathrm{BL}}^{+}(\operatorname{Re}, \operatorname{Pr})\right)+\alpha^{0.5} \beta^{-1} \operatorname{Re}^{0.1} \operatorname{Pr}^{0.5}\left|T_{b} / T_{w}-1\right|^{-1}} .
$$

Finally, in air, the following result is obtained for the relative variation of temperature:

$$
\frac{T_{\mathrm{rms}}^{\max }}{\bar{T}\left(y_{\mathrm{BL}}^{+}\right)}=\frac{2.5}{6.9 \operatorname{sign}\left(T_{b} / T_{w}-1\right)+5.8 \mathrm{Re}^{0.1}\left|T_{b} / T_{w}-1\right|^{-1}},
$$

where $\bar{T}^{+}\left(y_{\mathrm{BL}}^{+}=12\right) \approx 6.9$ is calculated from the formula proposed by Kader (1981). Equation (B 7) is plotted in figure 29 for several values of the ratio $T_{b} / T_{w}$ which is less (resp. greater) than unity in heating (resp. cooling) systems. The level of temperature fluctuations decreases with the Reynolds number in all cases and is larger in strongly heating and cooling systems. Using equation (B 7), the estimated levels of fluctuations in cases without radiation $\mathrm{A}, \mathrm{B}$ and $\mathrm{C}$ are $1.4 \%, 4.8 \%$ and $1.3 \%$ on the hot side, and $1.6 \%, 7.3 \%$ and $1.5 \%$ on the hot side, respectively. These numbers match fairly the results obtained in numerical simulations. Given the trends observed in figure 29, fluctuations of temperature can be expected to remain below $30 \%$ in most turbulent boundary layers in gaseous flows. Compressibility effects and viscous heating have here been neglected. 


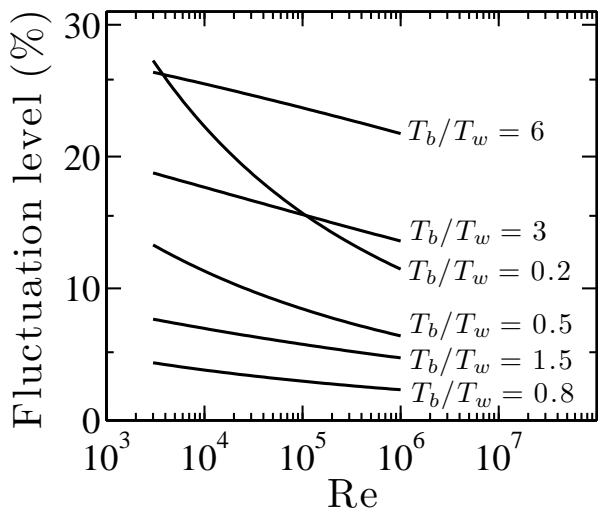

FIgURE 29. Relative variation of temperature $T_{\mathrm{rms}}^{\max } / T\left(y_{\mathrm{BL}}^{+}\right)$as a function of the Reynolds number for several values of $T_{b} / T_{w}$.

Abe, Hiroyuki, Kawamura, Hiroshi \& Matsuo, Yuichi 2004 Surface heat-flux fluctuations in a turbulent channel flow up to $R e_{\tau}=1020$ with $\operatorname{Pr}=0.025$ and 0.71. Int J. Heat Fluid Fl. 25, 404-419.

Abe, K., Kondoh, T. \& Nagano, Y. 1995 A new turbulence model for predicting fluid flow and heat transfer in separating and reattaching flows - ii. thermal field calculations. Int J. Heat Mass Transfer 38 (8), 1467-1481.

Ammouri, F. EI, Soufiani, A. \& TAine, J. 1994 Effects of temperature and concentrations in turbulent gas flows on combined radiative and conductive wall fluxes. In The Tenth International Heat Transfer Conference, , vol. 2. Brighton, UK.

Benarafa, Y., Clonia, O., Ducros, F. \& Sagaut, P. 2007 Temperature wall modelling for large-eddy simulation in a heated turbulent plane channel flow. Int J. Heat Mass Transfer 50 (21-22), 4360-4370.

Coelho, P. J. 2004 Detailed numerical simulation of radiative transfer in a nonluminous turbulent jet diffusion flame. Combustion and Flame 136 (4), 481-492.

Coelho, P. J. 2007 Numerical simulation of the interaction between turbulence and radiation in reactive flows. Prog. Energ. Combust. 33 (4), 311-383.

Coelho, Pedro J. 2012 Turbulence-Radiation Interaction: From Theory to Application in Numerical Simulations. J. Heat Trans.-T. ASME 134 (3).

Coelho, P. J., Teerling, O. J. \& Roeknerts, D. 2003 Spectral radiative effects and turbulence/radiation interaction in a non-luminous turbulent jet diffusion flame. Combust. Flame 133 (1-2), 75-91.

Coleman, GN, Kim, J \& Moser, RD 1995 A numerical study of turbulent supersonic isothermal-wall channel flow. J. Fluid Mech. 305, 159-183.

Dailey, LD, Meng, N \& Pletcher, RH 2003 Large eddy simulation of constant heat flux turbulent channel flow with property variations: Quasi-developed model and mean flow results. J. Heat Trans.-T. ASME 125 (1), 27-38.

Damien, Poitou, Jorge, Amaya, Mouna, El Hafi \& Benedicte, Cuenot 2012 Analysis of the interaction between turbulent combustion and thermal radiation using unsteady coupled LES/DOM simulations. Combust. Flame 159 (4), 1605-1618.

Debusschere, B. \& Rutland, C. J. 2004 Turbulent scalar transport mechanisms in plane channel and couette flows. Int J. Heat Mass Transfer 47 (8-9), 1771-1781.

Deshmukh, K. V., Haworth, D. C. \& Modest, M. F. 2007 Direct numerical simulation of turbulence-radiation interactions in homogeneous nonpremixed combustion systems. Proc. Combust. Inst. 31 (1), 1641-1648.

Deshmukh, K. V., Modest, M. F. \& Haworth, D. C. 2008 Direct numerical simulation of turbulence-radiation interactions in a statistically one-dimensional nonpremixed system. J. Quant. Spectrosc. Radiative Transfer 109 (14), 2391-2400. 
EDwARDs, D. K. 1976 Molecular gas band radiation, , vol. 12, pp. 115-193. New York: Academic Press, Inc.

Ghosh, S., Friedrich, R., Pfitzner, M., Stemmer, Chr, Cuenot, B. \& El Hafi, M. 2011 Effects of radiative heat transfer on the structure of turbulent supersonic channel flow. $J$. Fluid Mech. 677, 417-444.

Gore, J. P., Jeng, S.-M. \& Faeth, G. M. 1987 Spectral and total radiation properties of turbulent carbon monoxide/air diffusion flames. AIAA J. 25, 339-345.

Gupta, A., Haworth, D.C. \& Modest, M.F. 2013 Turbulence-radiation interactions in largeeddy simulations of luminous and nonluminous nonpremixed flames. Proceedings of the Combustion Institute 34 (1), 1281 - 1288.

Gupta, Ankur, Modest, Michael F. \& Haworth, Daniel C. 2009 Large-Eddy Simulation of Turbulence-Radiation Interactions in a Turbulent Planar Channel Flow. J. Heat Trans.T. ASME 131 (6).

Haworth, D. C. 2010 Progress in probability density function methods for turbulent reacting flows. Progress in Energy and Combustion Science In Press, Corrected Proof, - .

Huang, PG, Coleman, GN \& Bradshaw, P 1995 Compressible turbulent channel flows: DNS results and modelling. J. Fluid Mech. 305, 185-218.

JENG, S.-M. \& FAETh, G. M. 1984 Radiative heat fluxes near turbulent buoyant methane diffusion flames. J. Heat Transfer 106, 886-888.

KADER, B. A. 1981 Temperature and concentration profiles in fully turbulent boundary layers. Int J. Heat Mass Transfer 24, 1541-1544.

Kasagi, N., Tomita, Y. \& Kuroda, A. 1992 Direct numerical simulation of passive scalar field in a turbulent channel flow. J. Heat Transfer 114 (3), 598-606.

KaWAi, Soshi \& Larsson, Johan 2012 Wall-modeling in large eddy simulation: Length scales, grid resolution, and accuracy. Phys. Fluids 24 (1).

Kawamura, Hiroshi, Abe, Hiroyuki \& Matsuo, Yuichi 1999 Dns of turbulent heat transfer in channel flow with respect to reynolds and prandtl number effects. International Journal of Heat and Fluid Flow 20 (3), 196-207.

Kawamura, H, Ohsaka, K, AвE, H \& Yamamoto, K 1998 DNS of turbulent heat transfer in channel flow with low to medium-high Prandtl number fluid. Int. J. Heat Fluid Fl. 19 (5), 482-491.

KAYs, W. M. 1994 Turbulent Prandtl number. Where are we? J. Heat Trans.-T. ASME 116, $284-295$.

Kee, Robert J., Dixon-lewis, Graham, Warnatz, Jurgen, Coltrin, Michael E. \& Miller, James A. 1986 A fortran computer code package for the evaluation of gas-phase, multicomponent transport properties. Tech. Rep. SAND89-8009. Sandia National Laboratories.

Kee, R. J., Rupley, F. M. \& Miller, J. A. 1989 CHEMKIN-II: A Fortran Chemical Kinetics Package for the Analysis of Gas-Phase Chemical Kinetics. Tech. Rep. SAND89-8009. Sandia National Laboratories.

Kim, J. \& Moin, P. 1987 Transport of passive scalars in a turbulent channel flow. In Turbulent Shear Flows 6th, pp. 5-2.

Kong, Hojin, Choi, Haecheon \& Lee, Joon SiK 2000 Direct numerical simulation of turbulent thermal boundary layers. Physics of Fluids 12 (10), 2555-2568.

Larsson, J., Vicquelin, R. \& Bermejo-Moreno, I. 2011 Large eddy simulations of the hyshot ii scramjet. In Center for Turbulence Research Annual Briefs, pp. 63-74.

Lefebvre, H. \& Ballal, Dilip R. 2010 GAS Turbine Combustion, 3rd edn. Taylor and Francis Group.

LI, G \& Modest, MF 2003 Importance of turbulence radiation interactions in turbulent diffusion jet flames. J. Heat Trans.-T. ASME 125 (5), 831-838.

Moureau, V., Domingo, P. \& Vervisch, L. 2011a Design of a massively parallel CFD code for complex geometries. Compt. Rendus. Mec. 339 (2-3), 141-148.

Moureau, V., Domingo, P. \& Vervisch, L. $2011 b$ From large-eddy simulation to direct numerical simulation of a lean premixed swirl flame: Filtered laminar flame-pdf modeling. Combust. Flame 158 (7), 1340-1357.

NAGANO, Y. \& Kim, C. 1988 A 2-equation model for heat-transport in wall turbulent shear flows. Journal of Heat Transfer-Transactions of the ASME 110 (3), 583-589. 
Perrin, MY \& Hartmann, JM 1989 Temperature-dependent measurements and modeling of absorption by co2-n2 mixtures in the far line-wings of the $4.3-\mathrm{mu}-\mathrm{m} \operatorname{co} 2$ band. JOURNAL OF QUANTITATIVE SPECTROSCOPY \& RADIATIVE TRANSFER 42 (4), 311-317.

Piomelli, Ugo 2008 Wall-layer models for large-eddy simulations. Prog. Aerosp. Sci. 44 (6), 437-446.

Pope, S. B. 2000 Turbulent Flows. Cambridge University Press, New York.

Rivière, Philippe \& Soufiani, Anouar 2012 Updated band model parameters for h2o, co2, ch4 and co radiation at high temperature. International Journal of Heat and Mass Transfer 55 (13-14), 3349-3358.

Soucasse, L., Riviere, Ph. \& Soufiani, A. 2014 Subgrid-scale model for radiative transfer in turbulent participating media. JOURNAL OF COMPUTATIONAL PHYSICS 257 (A), $442-459$.

Soufiani, A. 1991 Temperature turbulence spectrum for high-temperature radiating gases. Journal of Thermophysics and Heat Transfer J. Thermophys Heat Tr. 5, 489-494.

Soufiani, A, Mignon, P \& Taine, J 1990 Radiation effects on turbulent heat transfer in channel flows of infrared active gases. In Proceedings of the 1990 AIAA/ ASME thermophysics and heat transfer conference, pp. 141-148.

Taine, Jean \& Soufiani, Anouar 1999 Gas IR Radiative Properties: From Spectroscopic Data to Approximate Models, , vol. Volume 33, pp. 295-414. Elsevier.

Tessé, Lionel, Dupoirieux, Francis \& Taine, Jean 2004 Monte carlo modeling of radiative transfer in a turbulent sooty flame. Int J. Heat Mass Transfer 47 (3), $555-572$.

Wu, Y., Haworth, D.C., Modest, M.F. \& Cuenot, B. 2005 Direct numerical simulation of turbulence/radiation interaction in premixed combustion systems. Proc. Combust. Inst. 30 (1), $639-646$.

Wu, Y., Modest, M.F. \& Haworth, D.C. 2007 A high-order photon monte carlo method for radiative transfer in direct numerical simulation. Journal of Computational Physics $223(2), 898-922$.

Zhang, Y. F., Vicquelin, R., Gicquel, O. \& Taine, J. $2013 a$ Physical study of radiation effects on the boundary layer structure in a turbulent channel flow. Int J. Heat Mass Transfer 61 (0), 654-666.

Zhang, Y. F., Vicquelin, R., Gicquel, O. \& Taine, J. $2013 b$ A wall model for les accounting for radiation effects. International Journal of Heat and Mass Transfer 67 (0), 712-723. 